\title{
○ 15. Seminar der LIBER Architecture Group
}

\author{
Inken Feldsien-Sudhaus
}

Die Nationalbibliothek in Madrid war die diesjährige gastgebende Institution für das 15. Seminar der LIBER Architecture Group vom 12. bis zum 17. April 2010. Das kombinierte Angebot von Besichtigungen und Vorträgen verbunden mit der Möglichkeit des Austausches fand wieder TeilnehmerInnen aus vielen Ländern Europas. Neben den klassischen Themen zu Fragen des Bibliotheksbaus beschäftigten sich auch einige Vorträge mit den Entwicklungen des Web und seiner Technologien sowie den Auswirkungen auf Serviceangebote der Bibliotheken. Aber auch Themen wie die Sanierung und Erweiterung von vorhandenen Gebäuden fanden ihren Raum. Die Tagung war perfekt von den spanischen Kolleginnen vor Ort organisiert.

This year the National Library of Spain in Madrid hosted the 15th Seminar of the LIBER Architecture Group from 12th to 17 th of April 2010. The innovative offer of tours and speeches as well as the possibility of program exchanges attracted interested participants from every corner of Europe. Of course, subjects such as renovation and extension of existing facilities abounded. This year to enhance more subjects of the day, several talks were directed towards the development of internet services within the library. These new internet technologies present interesting challenges for libraries wishing to implement these new programs. As expected, the Spanish team had organized the meeting in an excellent manner and every aspect of the conference was well thought through.

Fit for what purpose? Planning libraries for the users of the future

Ab $\bar{b} .1$ :

Spanische

Nationalbibliothek, Madrid, Außenansicht,

Foto: Feldsien
Die Nationalbibliothek von Spanien (Abb. 1) in Madrid war Gastgeberin des 15. Seminars der LIBER Architecture Group vom 12. April bis 17. April 2010. Bestandteil des Programms waren auch wieder Bibliotheksbesichtigungen vor und nach dem eigentlichen Vortragsprogramm. Das Interesse am Seminar war mit über 130 TeilnehmerInnen wieder hoch, das angebotene Besichtigungsprogramm am 12. und 13. April nahmen über 90 TeilnehmerInnen wahr.

Bibliotheken erleben zunehmend einen $\mathrm{Pa}$ radigmenwechsel. Neben klassischen The-

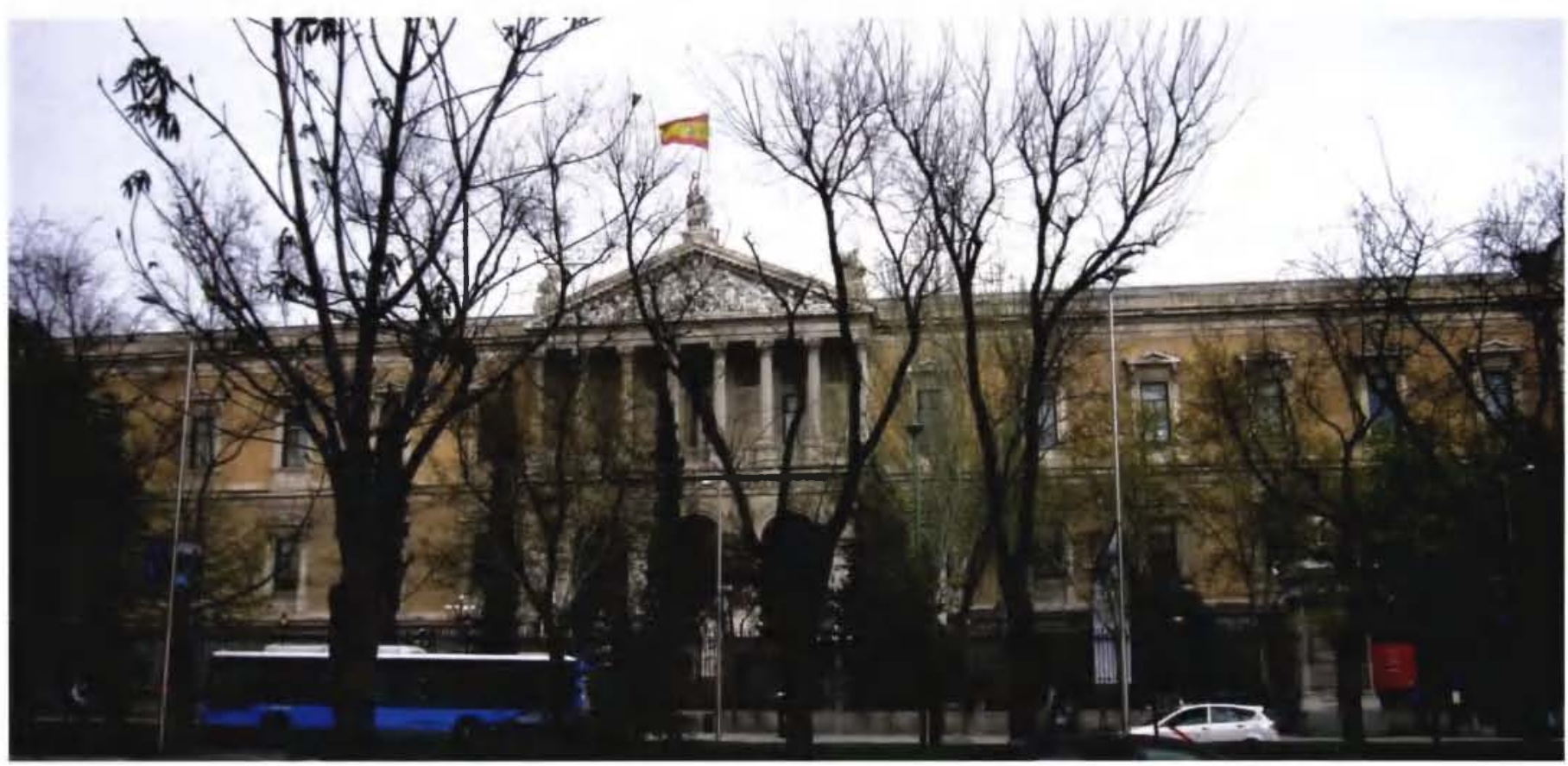


men des Bibliotheksbaus wurden auch Auswirkungen auf die Nutzung von Bibliotheksgebäuden in einigen Beiträgen der Tagung wie auch in den Pausen diskutiert. Web2.0 und neue Technologien dringen immer stärker in viele Lebensbereiche und in den Alltag der NutzerInnen vor. In diesem Beitrag wird auch auf verschiedene Videos, Studien und Links zusätzlich zu den eigentlichen Vortragspräsentationen hingewiesenen, die über die Internetseite des Seminars zugänglich sind und auf die hier nicht immer einzeln verwiesen wird.

Die Vulkanasche aus Island und das damit verbundene europaweite Flugverbot sowie der Eisenbahnerstreik in Frankreich verhinderte leider die Anreise eines Vortragenden verbündete jedoch die TagungsteilnehmerInnen, die sich zum Teil gemeinsam mit einem gemieteten Bus am Montag mittag dann auf die Heimreise machten.

\section{Setting the scene: \\ Learn - Experience - Meet}

Nach einem Überblicksreferat über das spanische Bibliothekswesen von Maria Antonia Carrato (Head of Library Coordination at the Ministry of Culture of Spain) hielt Wim de Waele (IBBT Research Institute; Gent) [1] das Einführungsreferat mit der Fragestellung, ob 13jährige Jungen als "digital Natives “ auch Bibliotheken noch schätzen werden.

Nach einer kurzen Zusammenstellung der Nutzungszeiten von PC, TV, Internet und Spielen seines 13 jährigen Sohnes wies er mit Bezug auf die Musikindustrie auf den rasanten Veränderungsprozess hin, von dem auch die Bibliotheken betroffen sind. Dazu forderte er, dass die öffentliche Hand sich stärker bei der Digitalisierung von gedruckten Werken engagieren sollte, ggfs. durch eine Kooperation mit privaten Firmen wie z. B. Google. Die Frage nach der Notwendigkeit eines gebauten Raumes für Bibliotheken in Zukunft beantwortete er mit einem klaren „Ja“, aber die Nutzung des Raumes wird sich stark verändern. Die Bibliothek wird dabei künftig zunächst ein Treffpunkt sein. Sie bietet dann Raum zum Lernen und Erfahren von Wissen und Kultur : Learn - Experience - Meet. Barrieren - räumlich wie auch virtuell - werden abgebaut, ähnlich wie im Kaufhaus.

Die technische Entwicklung befördert mobile Internet-Serviceangebote und eine Vernetzung der Beziehungen sozialer Kontakte, z.B. Facebook, Foursquare [2]. Das Analystenhaus Gartner zeichnet in verschiedenen Studien die Trends der ICT-Entwicklung (Information and Communication Technology) [3] nach. Bezogen auf die Entwicklung der mobilen Serviceangebote bis zum Jahr 2015 beziehen sich die Empfehlungen für Unternehmen und staatliche Einrichtungen auf folgende Themen:

- Bluetooth (3 and 4)

- The Mobile Web

- Mobile Widgets

- Platform-Independent Mobile AD Tools

- App Stores

- Enhanced Location Awareness (GPS-Nutzung, Datenschutz)

- Cellular Broadband

- Touchscreens

- M2M (Machine to machine)

- Device-Independent Security

Für Bibliothekskunden und Bibliotheken werden sich neue Partnerschaften ergeben, die kostenlosen Zugang zu Informationen bieten können. So ermöglicht eine Partnerschaft zwischen Foursquare und der "Financial Times" schon heute Studierenden von einigen Elite-Wirtschaftsuniversitäten den Zugang zu einem kostenlosen Abonnement, wenn sie sich in den Räumen einer Cafeteria der jeweiligen Universität in Foursquare einloggen [4]

Touchscreens werden sich gerade bei den Enduser-Geräten der Jugend massiv in den nächsten Jahren verbreiten und intuitives Verhalten erleichtern. Am MIT Media Lab's (Massachusetts Institute of Technology) wird von Pattie Maes [5] und Kollegen der "sechste Sinn" erforscht: ein integriertes, mobiles Gerät aus verschiedenen Komponenten mit Kamera, Projektor und Telefon. Das Gerät kann ohne Beschränkung eingesetzt werden, solange die Akkus funktionieren. Damit wird Interaktion überall möglich, auf jede Oberfläche kann projeziert werden. Metadaten bleiben aber der Schlüssel zur Information. Und der Wunsch nach Anleitung bzw. Unterstützung bei der Nutzung von Informationsquellen und Werkzeugen bleibt für etliche Nutzer von Bibliotheken oder Informationszentren weiterhin bestehen.

Zum Abschluss des Vortrags wurde das Projekt "de Krook" [6] vorgestellt. In der Stadt Gent mit zirka 250.000 Einwohnern sollen der alte Winterzirkus und ein Sportcenter für eine neue Stadtteil-Bibliothek, das Universitätsradio, ein multimediales Zentrum sowie für Bars und Restaurants umgenutzt werden. Am Projekt sind verschiedene Partner beteiligt: AG Stadsontwikkelingsbedrijf Gent (SOB), die Universität Gent und das Interdisciplinary Institute for Broad Band Technology (IBBT). Das Zentrum soll für das IBBT ein lebendiges Labor darstellen, zumal dort auch zentral für Belgien Multimedia-Material archiviert werden soll. Die Fertigstellung ist in fünf bis sechs Jahren geplant.

Deutlich wurde im Vortrag, dass Experimentieren der Schlüssel zur Zukunft ist. Die Bi-

\section{Seminar der LIBER Architecture Group}


- 15. Seminar der LIBER Architecture Group

\section{Abb. 2:}

National Technical Library: View on the Main Desk in the big Atrium (Level 2) Foto: Iveta Kopicova

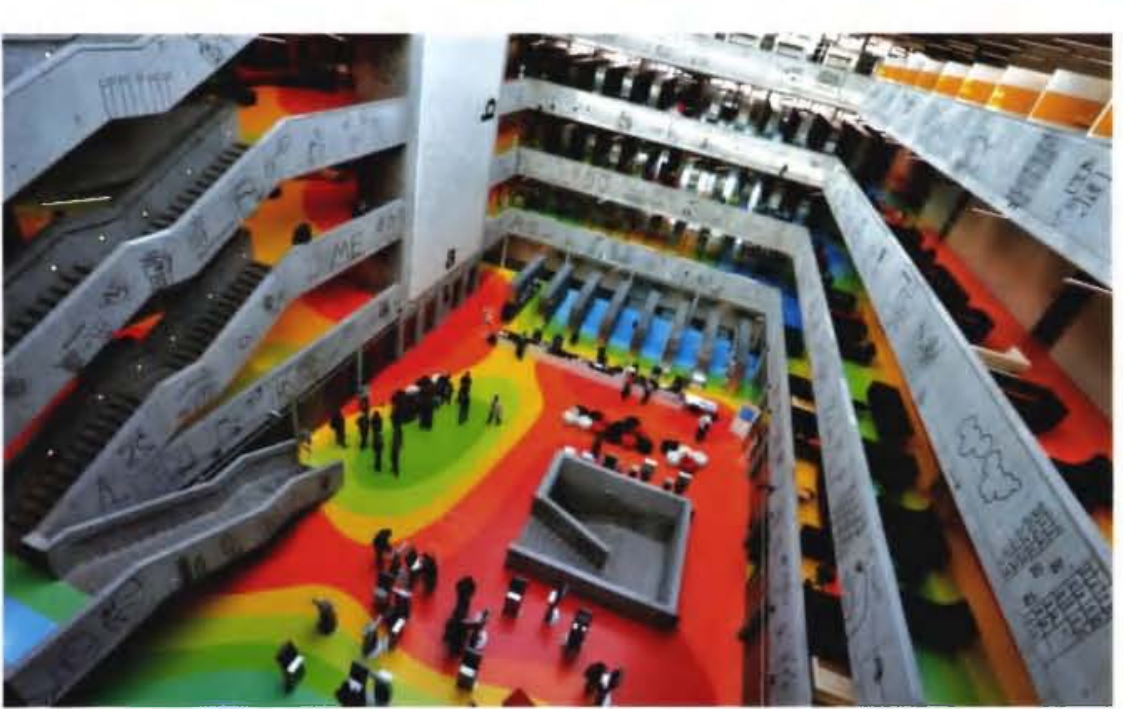

bliotheksnutzerInnen in den Städten, Kommunen und Hochschulen werden ihren eigenen Weg in die Zukunft genauso wie die unterschiedlichsten Serviceeinrichtungen wählen (müssen). Bezogen auf seinen eigenen Vortragstitel war das Fazit von Wim de Waele "Digital natives most likely will not love books but they might love libraries. " [7]

\section{Sustainable communities: Libra- ries in their local environment}

In Sevilla wird für die zentrale Universitätsbibliothek derzeit ein Gebäude - Biblioteca del Prado - von der Architektin Zaha Hadid erstellt. Über das Konzept des Gebäudes und die Überarbeitung des Wettbewerbentwurfs berichteten Sonsoles Celestino Angulo (Universitätsbibliothek Seville) und Andres Agudo Martínez (Architekt aus der Universitätsverwaltung). Zur Vorbereitung des Bauprojektes bereisten Mitarbeiter der Universität Europa und besichtigten schon durch Zaha Hadid erstellte Gebäude, so zum Beispiel das Phaeno in Wolfsburg. Durch Gerichte erlangten Anwohner im Moment einen temporären Baustopp.

Andre Hincker vom Strasbourg Public Library Network berichtet über die erfolgreiche Zusammenarbeit von 27 Partnern in dem 2001 gegründeten Netzwerk im Elsass [8] Es entstand während dieser Zeit z. B. die Mediathek "Andre Malraux" in Straßburg in einer alten Getreidelagerhalle am ehemaligen Flusshafen. Die Architekten sind JeanMarc IBOS and Myrto VITART [9]. Das Projekt umfasste zirka $12.000 \mathrm{~m}^{2}$ Nutzfläche, zirka 1.000 Benutzerarbeitsplätze, 750 Periodika im Freihandbereich, zirka $160.000 \mathrm{Me}$ dien im Freihandbereich sowie zirka 200.000 Bücher Altbestand und Inkunabeln.

Die „Technical University Library/Národni technickä knihovna (NTK)" [10] als "Gesamtkunstwerk" (Abb. 2) wurde durch Mar-

tin Svoboda aus Prag vorgestellt. Das Gebäude soll für angehende Architekten und Bauingenieure ein materialisiertes Lehrbuch darstellen, anhand dessen sie sich verschiedenste Lösungen anschauen und begreifen können. Die besondere Bedeutung eines guten Raumprogramms wurde als wichtige Grundlage für ein funktionierendes Gebäude herausgestrichen. Neben der Flexibilität wurde auch bei diesem Projekt hervorgehoben, dass das gegenseitige Vertrauen aller Planungspartner eine wichtige Grundlage für den Erfolg eines Projektes ist.

\section{Technical aspects of buildings}

In diesem Themenblock sollten Grundlagen der Haustechnik vermittelt werden:

- Prinzipien des Passivhauses (Design of the energy-efficient house: the Implications for libraries von Tönu Mauring, Tartu University)

( ) Lärmreduktion und -vermeidung (Acoustics von Gerard Le Goff, Architekt)

- Energieeinsparung bei der Klimatisierung (Energy restoration of the University and State Library Bremen and the impact on the user von Peter Ritzenhoff, Bremerhaven University of Applied Sciences)

Es gibt bisher nur wenige realisierte Beispiele von größeren Gewerbebauten, die dem Passivhaus-Standard entsprechen. Der Vortrag von Tōnu Mauring erläuterte, wodurch Energieverbrauch entsteht und in welchen Dimensionen Verbräuche reduziert werden. Im zweiten Vortrag versäumte es jedoch Gerard Le Goff, anhand der Umnutzung eines Industriebaus zugunsten einer Universitätsbibliothek in den Grands Moulins (Universite Paris Diderot, Paris 7) [11] Beispiele zum Thema Lärmreduktion und -vermeidung zu präsentieren. Dagegen wurde im dritten Vortrag wieder konkret auf die vorgenommenen baulichen Veränderungen und die positiven Auswirkungen in Bezug auf die finanzielle Ersparnis bei den laufenden Betriebskosten sowie die größere $\mathrm{Zu}$ friedenheit der NutzerInnen eingegangen.

\section{Changing and adapting library buildings}

Die gastgebende Bibliothek, die Nationalbibliothek von Spanien [12], residiert in Madrid in einem imposanten Gebäude aus dem Jahre 1896. Der Architekt Jerónimo Junquera [13] berichtete über seine Arbeiten, um diesen Altbau für aktuelle Bibliothekszwecke von 1987-2001 auszustatten und herzurichten. Zwei Jahre wurde an einem Masterplan für den Altbau in Madrid gearbeitet. Die Typologie des Gebäudes musste geändert werden, um heute die Bibliothek 


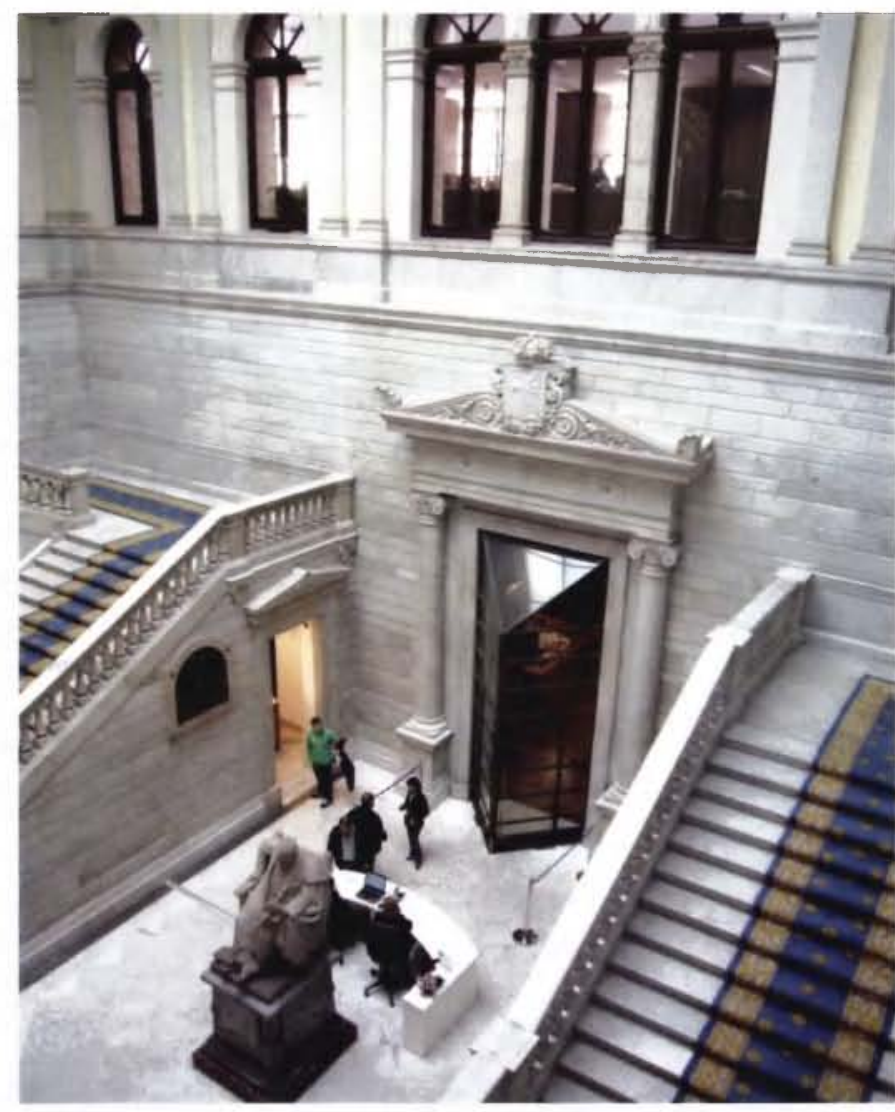

Abb. 3:

Spanische Nationalbibliothek, Madrid, Eingang, Foto: Feldsien

und das Museum der Bibliothek zu beherbergen (Abb. 3). Man entschied sich für eine neue Zonierung des Gebäudes. Hatte der Altbau zuvor zirka $21.800 \mathrm{~m}^{2}$, verfügt das Gebäude heute nach Umbau und Sanierung über $49.300 \mathrm{~m}^{2}$. Denkmalschutz wurde abgewogen gegenüber den Nutzeranforderungen, wie es auch bei anderen spanischen Projekten zu sehen war. Neue Elemente wurden hinzugefügt, damit das Gebäude nach heutigen Anforderungen genutzt werden kann (Abb. 4). Die Nationalbibliothek besitzt außerhalb von Madrid noch ein zweites Gebäude als Speichermagazin.

Über die Sanierung des Henry-van-de-Velde-Baus der Universitätsbibliothek Gent, und hier insbesondere über den Bücherturm, berichteten Sylvia van Peteghem, Chief Librarian, und Gert Jansseune in Vertretung des betreuenden Architektenbüros Robbrecht en Daem [14]. Bei dem Bücherturm, der auch unverändert weiter für diesen Zweck genutzt werden soll, wird aus bauphysikalischen Gründen eine zweite Haut (Box in der Box) eingezogen werden, um das Klima für die Bücher ohne Beeinträchtigung der originalen Außenhaut durch moderne Wärmedämm-Maßnahmen regeln zu können. Um Platz zu schaffen für die Bücher, die während der Sanierung aus dem Bücherturm ausgelagert werden müssen, wird eine neues unterirdisches Magaren gelegt.

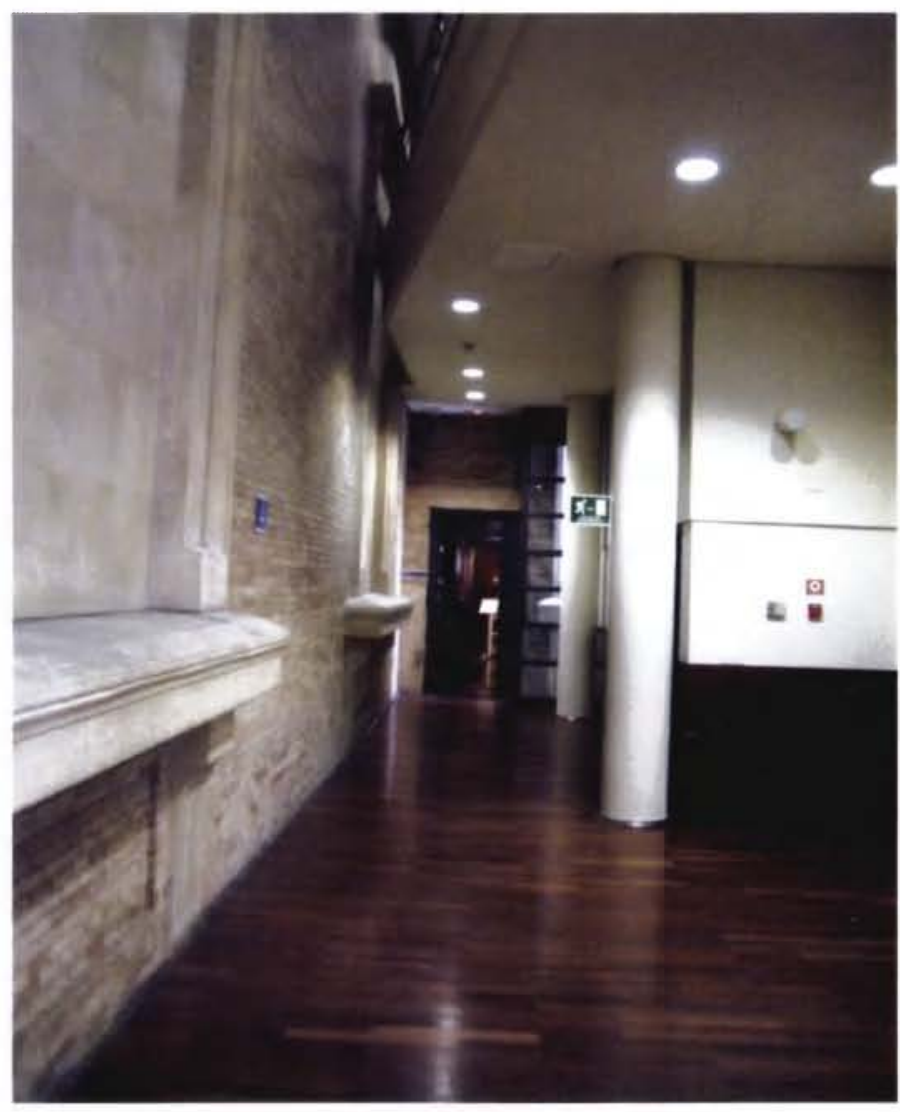

Abb. 4:

Nationalbibliothek, Madrid, Alt und neu, Foto: Feldsien

zin (acht Millionen Euro) gebaut, das sich beim Vergleich mit anderen Alternativen als beste Lösung in Bezug auf die entstehenden Kosten und Qualität der Lagerung des Altbestandes zeigte. Die oberste Etage des Bücherturms wird für verschiedenste Nutzungen wiederhergerichtet, bekommt einen 2. Lift und einen separaten Zugang. Aus Sicherheitsgründen dürfen allerdings nicht mehr als 50 Personen an Veranstaltungen teilnehmen. Große Sorgfalt wird bei diesem Projekt insgesamt auf die Wiederherstellung des originalen Zustandes aus den 30er Jah-

\section{New technology}

Nach der Vorstellung der Technischen Fachhochschule Wildau und ihrer Bibliothek beschäftigte sich Frank Seeliger mit dem Thema "Use of RFID Technology in Libraries: panacea or pain?". Er wies auf verschiedene technische Aspekte hin, die bei einer Einführung bekannt sein sollten

( ) die zur Zeit garantierte Haltbarkeit der so genannten Tags beträgt zehn Jahre;

() es treten immer wieder Probleme bei der Verarbeitung von Bücherstapeln auf;

( ) die Gates müssen einen Mindestabstand zu Metallregalen und anderen Metallteilen einhalten ${ }_{i}$ die Etiketten auf CDs, DVDs sind immer noch keine gute Lösung. 
- 15. Seminar der LIBER Architecture Group

Abb. 5:

Spanische

Nationalbibliothek,

Standort Alcalá

Foto: Feldsien

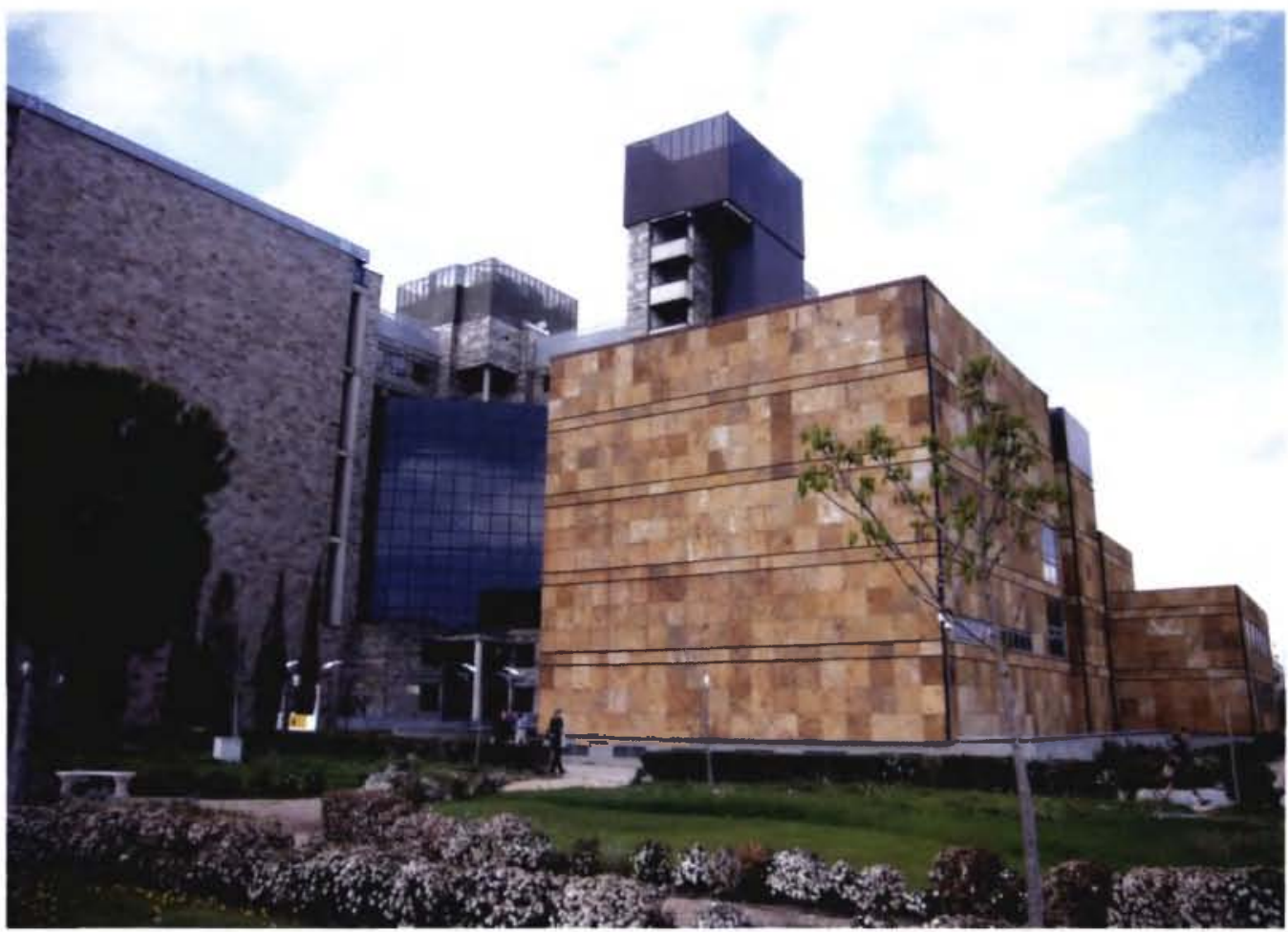

In seinem Vortrag zeigte Koen Rotteveel (DOK, Library Concept Center, Delft) unter dem Titel "Multitouch in the library - contextualization and visualization using Microsoft Surface" Beispiele für den Einsatz von multitouch screens in der Bibliothek, ein Mittel, um interaktiv, nicht-linear und einfach neue Inhalte zu entdecken und zu nutzen. Der Nutzer kann gemeinsam mit anderen Dinge gestalten, Einfluss nehmen oder sich informieren. In der Stadtbibliothek Delft wurden z. B. 25.000 Fotos von Leuten, Straßen und Ereignissen in Delft digitalisiert und katalogisiert. Auf diese Fotos kann einfach über den Bibliotheksausweis und den Microsoft "Tisch" über die Postleitzahl auf die Fotos der eigenen Wohnstraße zugegriffen werden [15]. Der Spassfaktor dabei ist groß! Nicht zu vergessen ist dabei, dass diese Technologie Geld kostet.

\section{Joint-use libraries}

Unter diesem Thema wurden drei völlig unterschiedliche Projekte vorgestellt. Nach zehn Jahren im Neubau auf dem Viikki Campus in Helsinki wurde die Campusbibliothek evaluiert. Flächen und Einrichtungen werden heute gemeinsam mit einer Stadtteilbibliothek betrieben. Es sind im Moment noch zwei getrennte Bibliotheken, wenn auch die Entwicklung offen ist. Das "Info Centre Korona - the heart of the Viikki campus" gab sogar lt. Marja Moisio, (Helsinki University Library, Viikki Campus Library) nicht mehr benötigte Flächen an die Universität zurück.

Die Sanierung und Umbau des früheren Gebäudes Richelieu der französischen Natio- nalbibliothek stellt ein großes Projekt dar, dazu soll das Gebäude von drei unterschiedlichen Institutionen genutzt werden. Marie de Laubier (Bibliotheque nationale de France) gibt für die drei Institutionen $\mathrm{BnF}$, l'Institut National d'Histoire d'Art und Ecole Nationale des Chartes einen Überblick über die neue interne Aufteilung des Gebäudes. Die Fertigstellung ist für 2017 geplant und beinhaltet neben einem neuen Eingang auch die Einhaltung aktueller Brandschutzbestimmungen. Die von der Sanierung und den Umbauten betroffenen MitarbeiterInnen werden vorübergehend in Containern untergebracht. Eine echte Integration der drei Einrichtungen ist zum jetzigen Zeitpunkt nicht geplant.

Dies sieht beim dritten Projekt völlig anders aus: Dr Judith Keene (University of Worcester) stellte das Worcester Library and History Centre vor. Hier werden unter anderem die Stadtbibliothek und die Universitätsbibliothek mit dem Archiv und dem zentralen Anlaufpunkt der Stadtverwaltung ("HUB") zusammengelegt. Gut zwei Jahre hat der Prozess des gedanklichen Zusammenwachsens für die Bibliotheken gedauert. Insgesamt werden die Bürger und Nutzer auf breiter Ebene in den Planungsprozess eingebunden. Die Firma GallifordTry [16] gewann die PPP-Ausschreibung für das Projekt. Als Architekten sind Feilden Clegg Bradley Studios engagiert. Die Zonierung innerhalb des Gebäudes erfolgt nach Aktivitäten, nicht nach Inhalten bzw. Materialien. Der Neubau soll im Sommer 2012 eröffnet werden und erhält in den "Excellent" gemäß der Building Research ökologischen Standards die Bewertung 
Establishment Environmental Assessment Method (BREEAM) [17]

\section{National Library of Spain}

Die Nationalbibliothek nutzt zwei Häuser. Der Architekt Francisco Longovia präsentierte das Speichergebäude "The Alcalá de Henares building project of the National Library of Spain" (Abb. 5). Neben der erforderlichen Speicherkapazität wird in dem außerhalb Madrids gelegenen Gebäude auch ein kleiner Lesesaal angeboten, was für die in Alcalá ansässige Universität von besonderem Nutzen ist. Aber auch die Restaurierung von Plakaten und anderen Materialien sowie die Katalogisierung erfolgt dort direkt vor Ort. Der Standort wurde in mehreren Bauabschnitten (1985 bis 2010) ausgebaut, dabei ist der Grundriss der Büchertürme jeweils quadratisch. Die jüngste Erweiterung umfasst auch ein robot-gesteuertes Hochmagazin (Abb. 6) für insgesamt zwei Millionen Medien.

\section{Long-term storage of collections}

Am Freitag wurden durch zwei Vorträge anschaulich physikalische Grundlagen zur Planung von Räumen für die Langzeitarchivierung vermittelt.

"Long term storage in low temperature stacks" von Marie Vest (Royal Library, Copenhagen) stellt anschaulich die Überlegungen der Kollegen der Königlichen Bibliothek zum notwendigen Kompromiss in Bezug auf das optimal einzuhaltende Klima und Feuchtigkeit für die verschiedensten Materialien dar. Die Königliche Bibliothek versorgt heute auch die Universität mit. Die Erweiterung der Humanistischen Fakultätsbibliothek (2005-2008) in der Njalsgade 112 (Architekten Dissing und Weitling) [18] schaffte auch zusätzlichen Speicherraum für die Königliche Bibliothek. Es wurde deutlich, dass Zerfallsprozesse der verschiedenen Materialien von Medien durch richtige Lagerhaltung signifikant verzögert werden können und damit eine auf das jeweilige Material bezogene Klimatisierung auch wirtschaftlich effizient sein kann. In diesen Kalt-Magazinen lagern vorrangig wenig genutzte Pflichtexemplare, die im Falle einer Benutzung nicht zur sofortigen Ausleihe, sondern mit einem Zeitverzug bereitgestellt werden.

Unterschiedliche Aspekte zeigte Deborah Novotny (Head of Preservation, British Library) in „Fit for purpose: British Library Storage. Buildings for the future". In London wurde eine Erweiterung des Britischen $\mathrm{Na}$ tionalbibliothek um das British Library Centre for Conservation (BLCC) gebaut (2005-
2007). Weiterhin wird eine Erweiterung in Boston Spa errichtet; Architekten Atkins Design Studio [19]. Auch hier wird ein automatisiertes Hochregallager eingebaut. Besondere Aufmerksamkeit wurde auf den Brandschutz gelegt. Die Klimatisierung wird mit einer Low-Oxygen-Anlage [20] erfolgen, der Sauerstoffanteil liegt bei nur zirka 15 Prozent. Der Mensch kann dann noch mit Mühe - wie in den hochalpinen Bergen - atmen, für die Entstehung von Bränden ist hingegen der Sauerstoffanteil in der Luft zu gering. Regulär sollte dieser bei 19,5 Prozent (z. B. US-amerikanischer Standard OSHA) liegen. Für die Zeitschriften plant die British Library auch ein neues Magazin (Colindale Newspaper Library). Für die Lagerung von Mikrofilmen will die Bibliothek mit einer Privatfirma (Vindon Scientific Ltd, Rochdale, Lancashire) zusammenarbeiten, die unter anderem für die Pharmaindustrie Kühlhauskapazitäten bereitstellt.
15. Seminar der $\odot$ LIBER Architecture Group

Abb. 6:

Spanische

Nationalbibliothek, Standort Alcalá, Automatisiertes

Magazin,

Foto: Feldsien

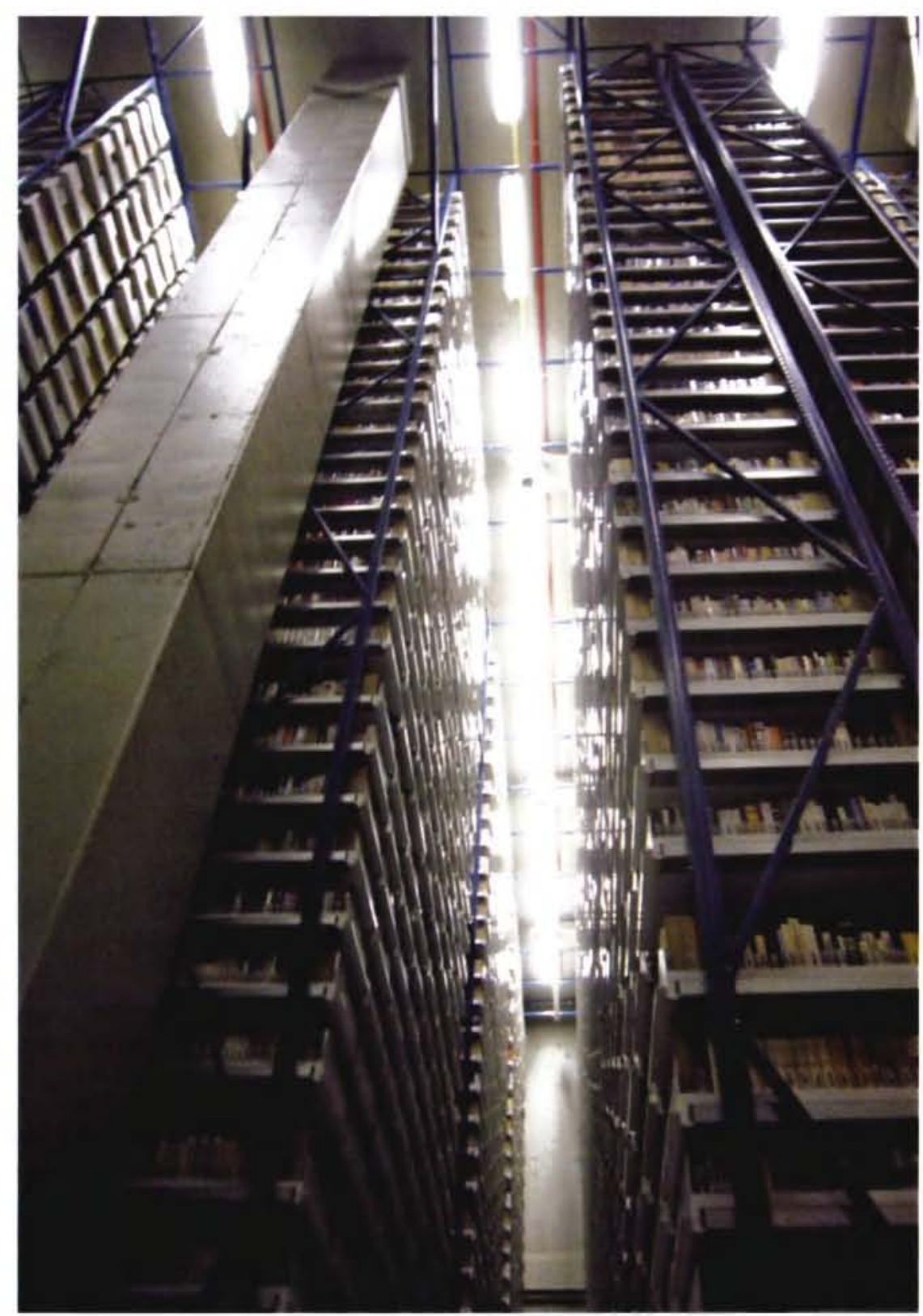


( ) 15. Seminar der LIBER Architecture Group

\section{Refurbished buildings}

Über die Sanierung und Erweiterung der Universitätsbibliothek, der David Wilson Library [21], berichtete Christine Fyfe (University of Leicester). Die Bibliothek wurde Ende der $60^{\prime}$ er Jahre geplant und 1974 eröffnet. Nach 30 Jahren wies das Gebäude die üblichen Mängel auf: überfüllt, kein Fenster zu öffnen, kein natürliches Licht, die Technik und die Raumaufteilung nicht mehr den heutigen Anforderungen entsprechend.

Die Planungen ergaben dann, dass es kostengünstiger ist, am Standort zu sanieren und zu erweitern. Ziele waren dabei neben der stärkeren Nutzung des natürlichen Lichts, die Entstehung eines angenehmen Ambientes und eine übersichtliche Raumaufteilung Dazu wurde ein neuer Eingang geschaffen Die Erweiterungsflächen im Publikumsbereich sind für Kompaktregalanlagen geplant. Im Untergeschoss der Erweiterung wurden zudem ein Hörsaal mit 500 Plätzen sowie zwei Seminarräume erbaut

Die TU-Bibliothek in Delft war vielen SeminarteilnehmerInnen bekannt, da das LAG-Seminar 2006 dort schon zu Gast war. Wilma van Wezenbeek kreierte mit ihrem Titel TU Delft Library Educate Innovate Create fast schon ein weiteres Motto für das Seminar. Auch hier überlegen Kollegen, welche Rolle künftig eine Bibliothek in einer technischen Universität übernehmen kann. Die Universität plante bereits ein separates Learning Center ohne Beteiligung der Bibliothek. Das war eine Herausforderung für die Bibliothek. In Workshops und in Interviews wurden durch Projektgruppen die Bedürfnisse der verschiedenen Nutzergruppen erforscht, die idealerweise der Bibliothek ihr Leben lang verbunden bleiben In Konkurrenz zu anderen, aufwändigen Projekten innerhalb der Universität versucht die Bibliothek, möglichst zügig und schnell Studierenden eine verbesserte Infrastruktur und eine flexiblere Lernumgebung in Wohnzimmeratmosphäre anzubieten. Vier Projekträume wurden mit Fototapete individualisiert: Europa, Asien, Amerika und Afrika. Die Nachfrage nach Arbeitsplätzen konzentrierte sich bisher in Delft auf die Prüfungswochen. Künftig gelten in den Examenszeiten andere Benutzungsregeln als während des laufenden Semesters. Im neuen Library Learning Center sind Telefonate, Kaffee trinken und Essen sowie Reden erlaubt. Nur zu den Prüfungszeiten wird das Reden und Telefonieren verboten. Die Bibliothek hat sich vorgenommen, auch Events anzubieten: Ausstellungen, Lesungen, Treffpunkt. Die neue Einrichtung ist mobil und flexibel. Die Bibliothek sieht sich auch in der Rolle des "knowledge advisor" mit einem live chat. Es gibt aber auch gezielte Angebote, z. B. bei Problemen mit Mechanik und Mathematik können sich
Studierende innerhalb einer bestimmten Frist an die Information in der Bibliothek wenden oder per Skype Fragen stellen. Dieses ist eine Gemeinschaftsaktion der Bibliothek, der Studierendenschaft und der Universitätsverwaltung (Education \& Student Affairs).

Über den langwierigen Entscheidungs- und Finanzierungsprozess zur Sanierung der Bibliotheque Interuniversitaire de la Sorbonne berichtete Philippe Marcerou (Bibliotheque Interuniversitaire de la Sorbonne). Aufgrund der veralteten Technik und nicht mehr zugelassenen Sicherheitseinrichtungen war eine Sanierung unumgänglich geworden. Während dieser Zeit ist die Bibliothek in ein anderes Gebäude innerhalb von Paris umgezogen. Die Bauarbeiten sollen von Juli 2010 bis März 2013 durchgeführt werden.

\section{Collaboration between librarians and architects}

In seinem Vortrag "No fairy tale: The Jacobund-Wilhelm-Grimm-Zentrum [in Berlin] successful collaboration between architects and librarians" betonte Olaf Eigenbrodt (seit 2010 Universität Hamburg) die Bedeutung der vertrauensvollen und verantwortungsbewussten Zusammenarbeit zwischen den verschiedenen Projektbeteiligten. Bei diesem Projekt wurde von den Architekten (Max Dudler) [22] und Planern die Humboldt-Universität, vertreten durch die Technische Abteilung, den Computer- und Medienservice und die Universitätsbibliothek, als Kunde wahrgenommen. Die Planer gingen gemeinsam auf Besichtigungstour. Dabei wurde eine solide Grundlage für die spätere Zusammenarbeit gelegt, die auch Konflikte bewältigen half. Heute zeigt das neue Gebäude eine hohe Akzeptanz durch die NutzerInnen (5.000 Besucher/Tag). Diese Nachfrage bereitet aber auch Probleme, z. B. eine zu enge Treppe ins Untergeschoss zu den Garderoben sowie die zu geringe Anzahl der Garderobenschränke.

Die Zuhörer lernten am Freitag durch den Vortrag des Architekten Jose Maria Peridis "A Library in the skyline of Toledo" die Biblioteca de Castilla La Mancha [23] kennen, die am Sonnabend besichtigt werden konnte. Deutlich wurde dabeì die besondere Bedeutung des Ortes aus der Zeit des spanischen Bürgerkriegs. Die Bibliothek ist Teil der Festung Alcázar, die im Bürgerkrieg stark zerstört und danach rekonstruiert wurde. Die Bibliothek wurde 1998 in dem Gebäude eröffnet. Sie bietet einen großen, zentralen Lesesaal, aber auch einen besonders schönen Ausblick auf die Stadt Toledo. Das historische Gebäude prägt mit seinen dicken Mauern natürlich die Räume der Bibliothek. 


\section{Besichtigungen [24]}

Das Bibliothekssystem von Madrid [25] umfasst neben der Regionalbibliothek derzeit zirka 230 Bibliotheken, davon 13 Bücherbusse und 12 Bibliometro's:

- 16 Bibliotecas Públicas

(insgesamt 1.249.916 Medieneinheiten, 2.160 Leseplätze, 447 Leseplätze Zeitungen, 299 Internetarbeitsplätze)

( 133 Bibliotecas municipales

- 13 Bibliobuses

○ 75 Centro de Lectura

- Bibliotecas Escolares

Biblioteca Pública Municipal Usera 'Jose Hierro', Madrid

$4.138 \mathrm{~m}^{2}, 1995$ bis 2002, Eröffnung Oktober 2003 [26]

Adresse: Avenida Rafaela Ybarra, 43.

28026 Madrid

Metro: Plaza Elíptica

Architekten:

Inaki Ábalos und Juan Herreros [27]

Leseplätze: 256, Multimediaarbeitsplätze: 46, Audio-Abspielplätze: sechs

Die Bibliothek (Abb. 7), ein erster Preis eines Architektenwettbewerbes, liegt im neuen Zentrum benachbart zum Rathaus. Das sieben Stockwerke hohe Gebäude wird charakterisiert durch feststehende Verschattungselemente, die an der Fassade wie ein geöffnetes Buch erscheinen. Jedes Stockwerk hat zur leichteren Orientierung eine eigene Farbe. Die Tapeten sind ein Entwurf des Künstlers Peter Halley [28]. Die Lesebereiche wirken durch die hohen Decken großzügig (Abb. 8). Seitlich sind auf Galerien Gruppenarbeitsräume bzw. ein Minikino für Kleingruppen angeordnet (Abb. 9). Ein großer Raum für Veranstaltungen wird leider nicht genutzt, da aufgrund der gewählten harten Materialien der Nachhall im

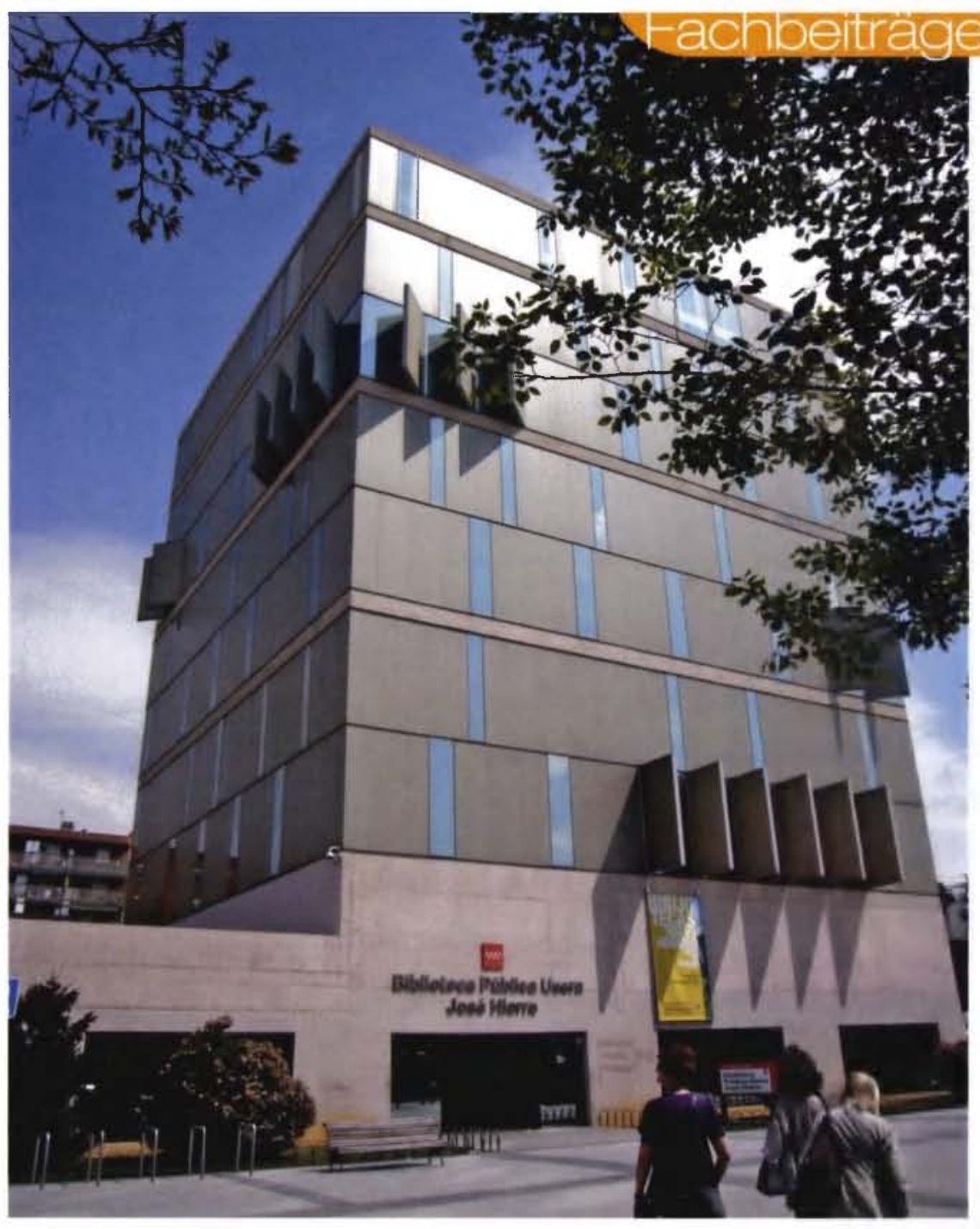

Abb. 7: Biblioteca Pública Municipal Usera 'Jose Hierro', Ansicht, Foto: Feldsien

Raum zu groß ist. Hier sind Nachrüstungen schwierig, da insbesondere die extra gestalteten Tapeten als Kunst nicht einfach durch schallschluckende Elemente ersetzt werden können ohne den Charakter des Raumes zu zerstören. Schade. Geplant ist das Gebäude für zirka 81.000 Medien.

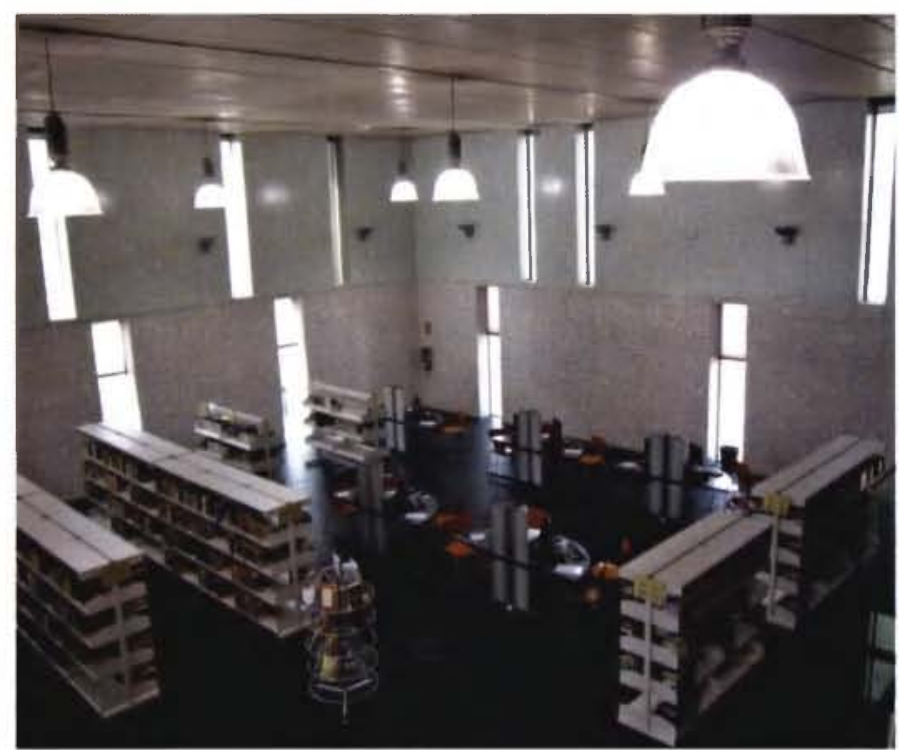

Abb. 8: Biblioteca 'Jose Hierro' Lesesaal, Foto: Feldsien

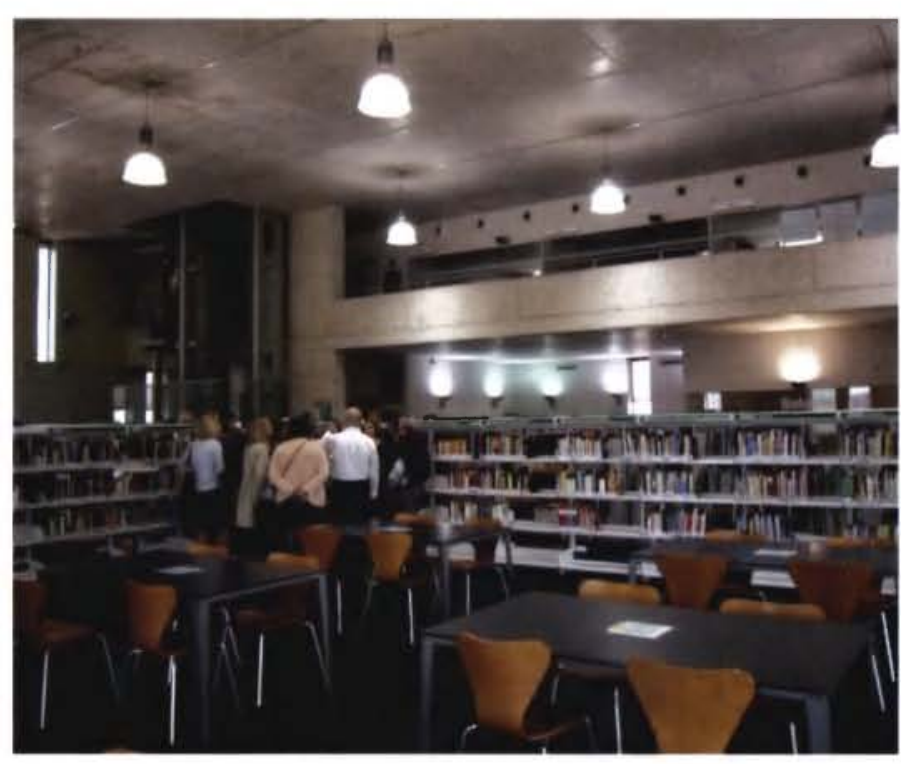

Abb. 9: Biblioteca 'Jose Hierro'Galerie, Foto: Feldsien 


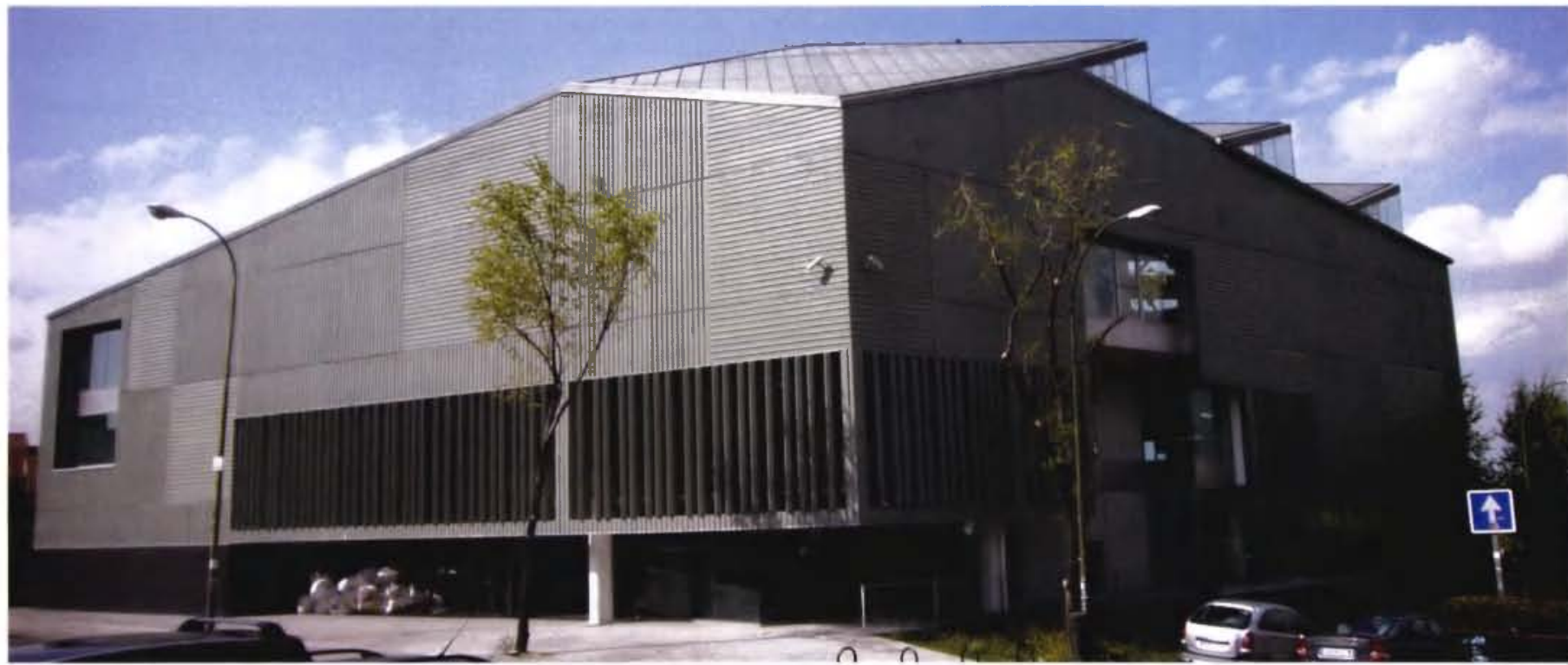

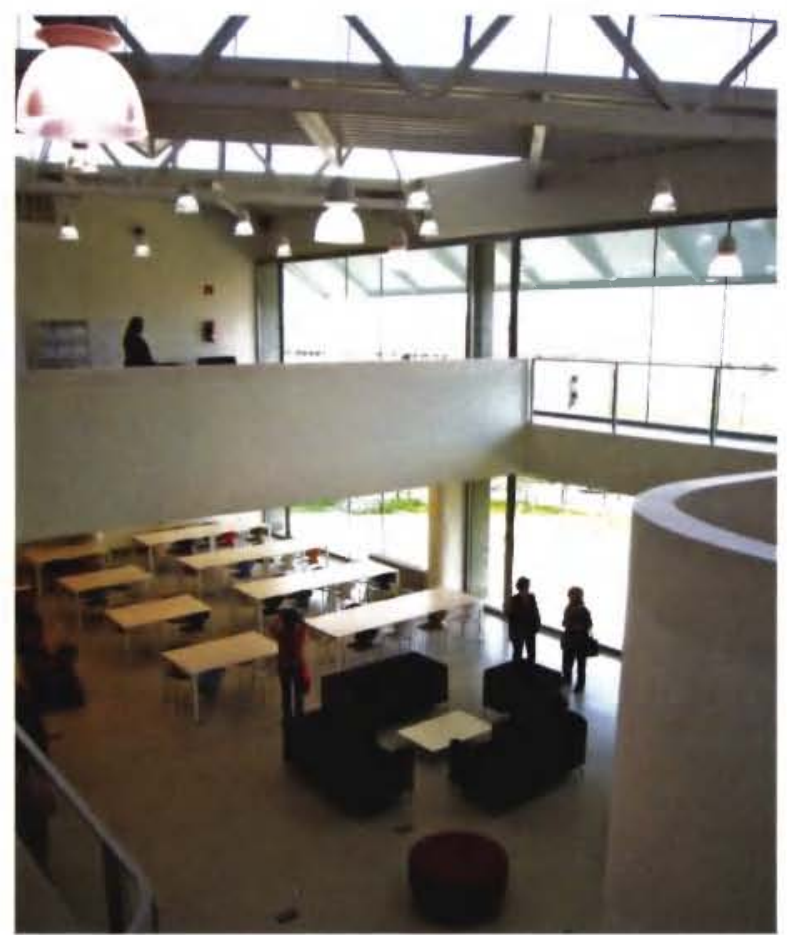

Abb. 10 (oben): Biblioteca Publica

Municipal de Carabanchel Ansicht

Foto: Feldsien

Abb. 11:

Biblioteca de

Carabanchel, Blick über verschiedene Ebenen,

Foto: Feldsien
Biblioteca Pública Municipal de Carabanchel, Madrid

Zirka $4.800 \mathrm{~m}^{2}$, Eröffnung im Mai 2010 (Abb. 10) Architekten: Pau Soler Serratosa und Miguel Rodríguez Gonzalez

Die Bibliothek ist offen gestaltet (Abb. 11) und soll die Selbstbedienung des Nutzers mit unterstützen. Dazu wird auch RFID eingesetzt werden. Die Lage des Gebäudes am Hang bietet einen wunderbaren Blick über Madrid (Abb. 12). Hier wäre auch ein kommerzielles Cafe gut vorstellbar oder wenigstens mehr Sitzplätze mit Ausblick.

\section{Bibliometro (29)}

In 12 Metro-Stationen (Abb. 13) (Aluche Embajadores; Canal; Carabanchel Alto; Legazpi; Mar de Cristal; Moncloa; Sierra de Guadalupe; Puerta de Arganda; Nuevos Ministerios; Puerta del Sur; Chamartin) gibt es kleine Bibliotheken, die montags bis freitags von 13.30 bis 20.00 Uhr geöffnet sind.

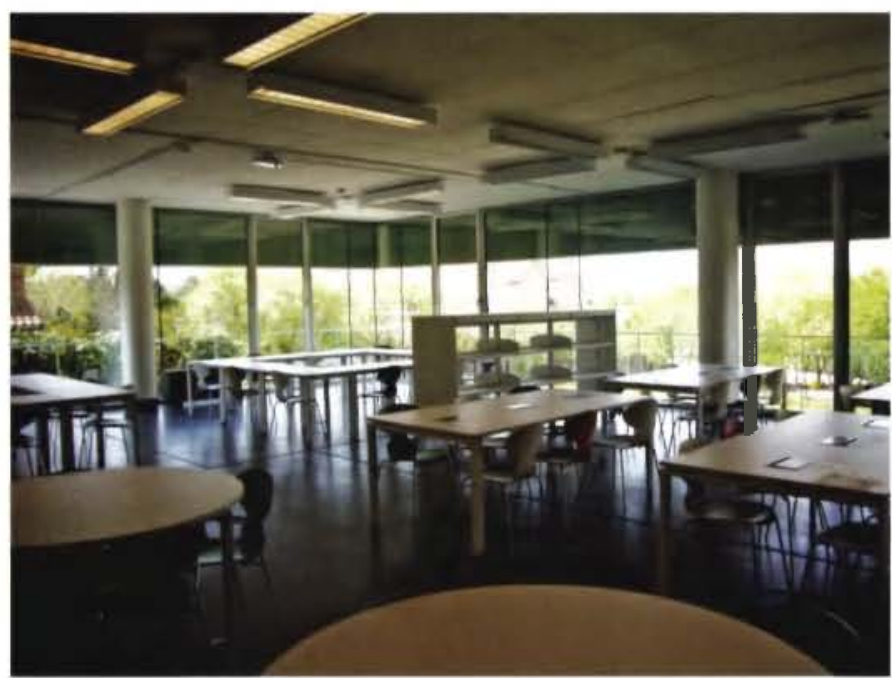

Abb. 12: Biblioteca de Carabanchel, Lesebereich, Foto: Feldsien

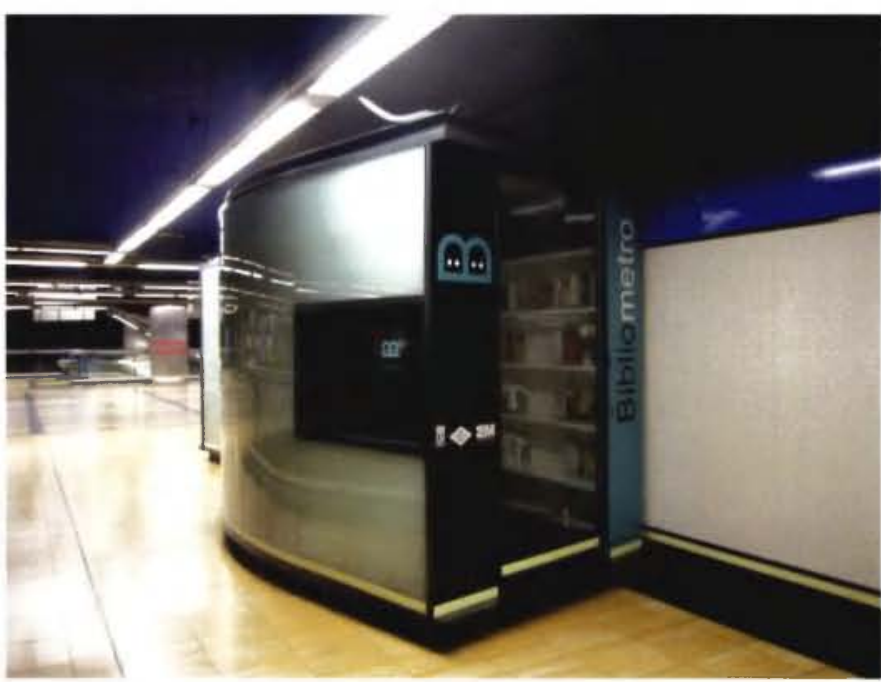

Abb. 13: Bibliometro, Station Chamartin Foto: Feldsien 


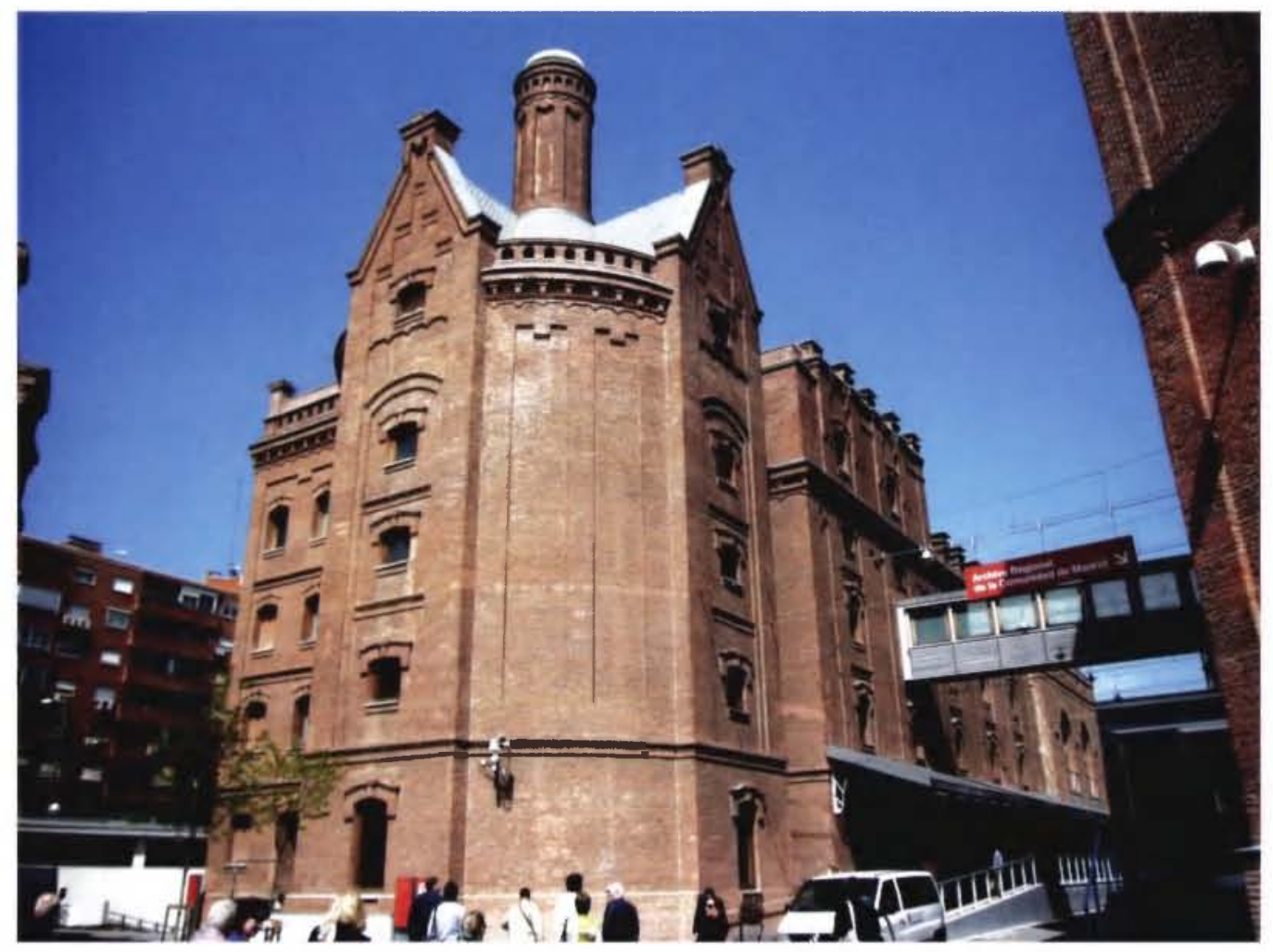

\section{Seminar der $\odot$ LIBER Architecture Group}

Abb. 14 (links):

Biblioteca Regional de

Madrid 'Joaquin Leguina', Ansicht,

Foto: Feldsien

Abb. 15 (unten rechts):

Biblioteca 'Joaquin

Leguina', Leseplätze im historischen Ambiente,

Foto: Feldsien

Abb. 16 (unten links): Biblioteca 'Joaquin Leguina', Abspielplätze, Foto: Feldsien

Biblioteca Regional de Madrid 'Joaquin Leguina', Madrid [30] Zirka $10.000 \mathrm{~m}^{2}, 1997$ bis 2002 Adresse : Calle Ramírez de Prado, n 3. 28045 Madrid

Metro: Delicias (L3) und Mendez Álvaro (L6) Architekten: Luis Moreno Garcia-Mansilla und Emilio Tunon

Leseplätze: 152, Internet/OPAC-Arbeitsplätze: 94

Diese Regionalbibliothek ist für Madrid die Pflichtexemplarbibliothek und damit eine Präsenzbibliothek. Als Gebäude [31] wurde eine ehemalige Bierbrauerei, El Águila (Baujahr 1912 bis 1914), umgenutzt (Abb. 14). Alte technische Einrichtungen wie Silos wurden z. T. erhalten und in die Bibliothek integriert (Abb. 15). Neue Einbauten sind z. T. farbig abgesetzt (Abb. 16). Gegenüber der Bibliothek gelegen ist das Archivo Regional de Madrid.
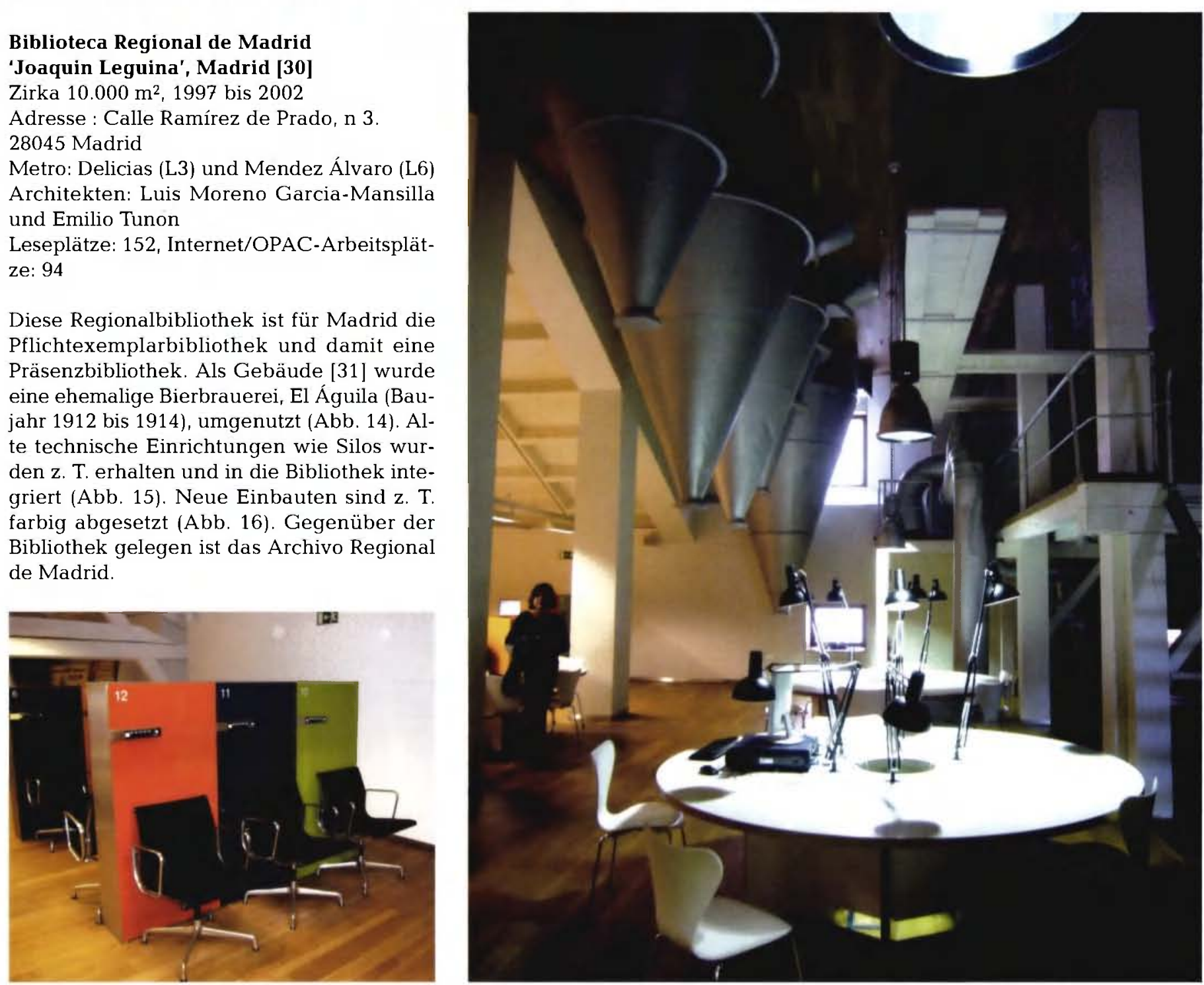

10.1515/ABITECH.2010.30.2.100 
○ 15. Seminar der LIBER Architecture Group

Abb. 17:

UNED

Universitätsbibliothek, Ansicht,

Foto: Feldsien

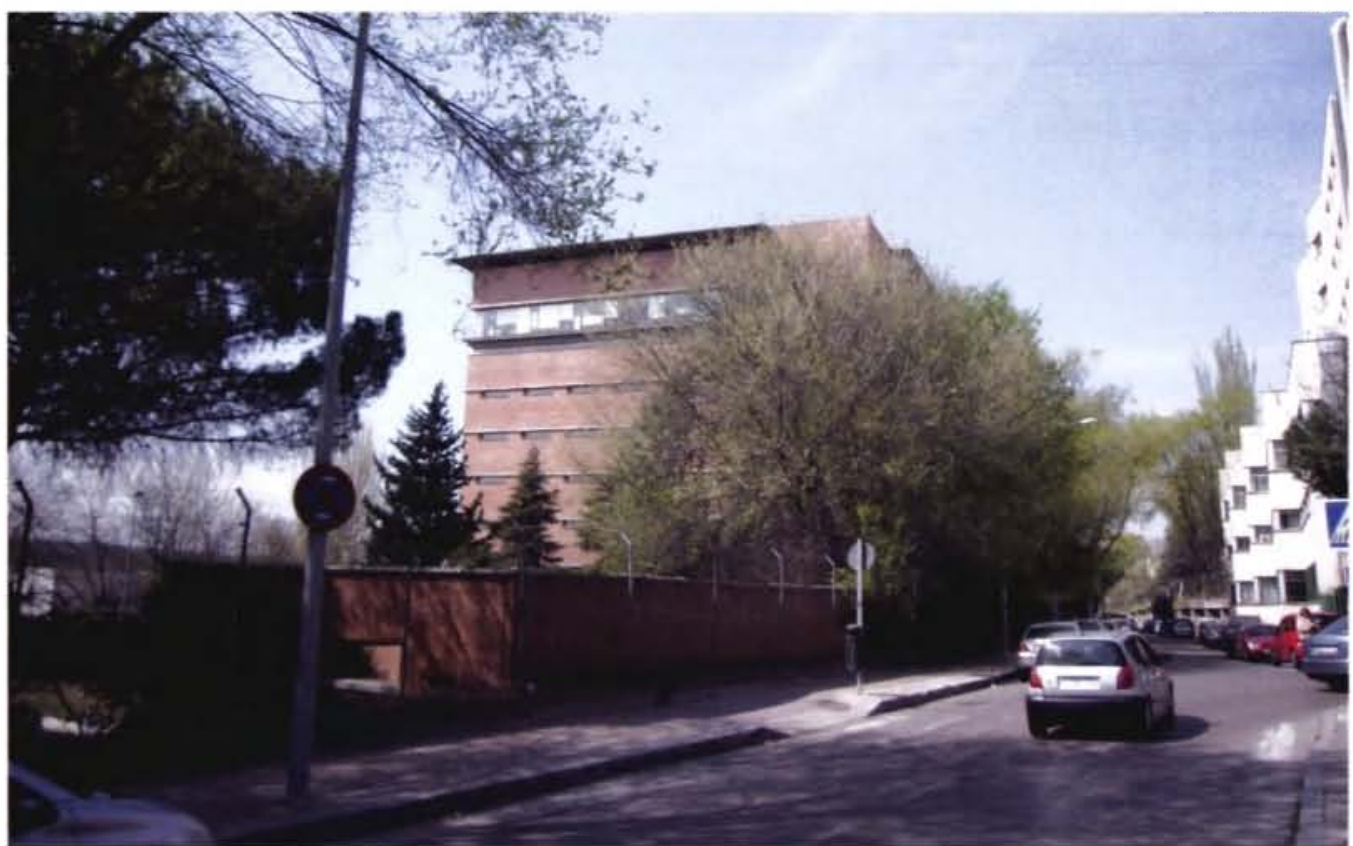

UNED University Library (Universidad Nacional de Educacion a Distancia), Madrid [32]

Zirka $10.000 \mathrm{~m}^{2}$, Baujahr 1989 bis 1994 Architekt Jose I. Linazasoro,

Adresse : Po Senda del Rey, 5. 28040-Madrid Metro: Metro Línea 6 Ciudad Universitaria (L6)

Leseplätze: zirka 450

Die UNED ist eine Fern-Universität, bei der die Studierenden zu Prüfungszeiten persönlich erscheinen. Das Bibliotheksgebäude ist umgeben von lauten Straßen, daher präsentiert sich das Gebäude mit einer geschlosse- nen, gemauerten Fassade (Abb. 17). Der Charakter der Lesesäle wird bestimmt durch ein rundes Atrium, das sich über alle LeseGeschosse erstreckt. Damit wird Licht in den Innenraum des Gebäudes durch Oberlichter geführt. Arbeitsplätze auf den Etagen sind mit Ausblick auf das Atrium angeordnet (Abb. 18). Als Kontrast dazu ist die Cafeteria voll verglast, um den schönen Ausblick in die Landschaft genießen zu können. Die Eingangsebene ist von den Lesesälen getrennt, damit wird der Lärm aus der Ausleihe und dem Eingangsbereich auch auf die Eingangszone beschränkt. Das Gebäude beinhaltet zirka 420.000 Medien.
Abb. 18:

UNED

Universitätsbibliothek, Atrium und Leseplätze, Foto: Feldsien

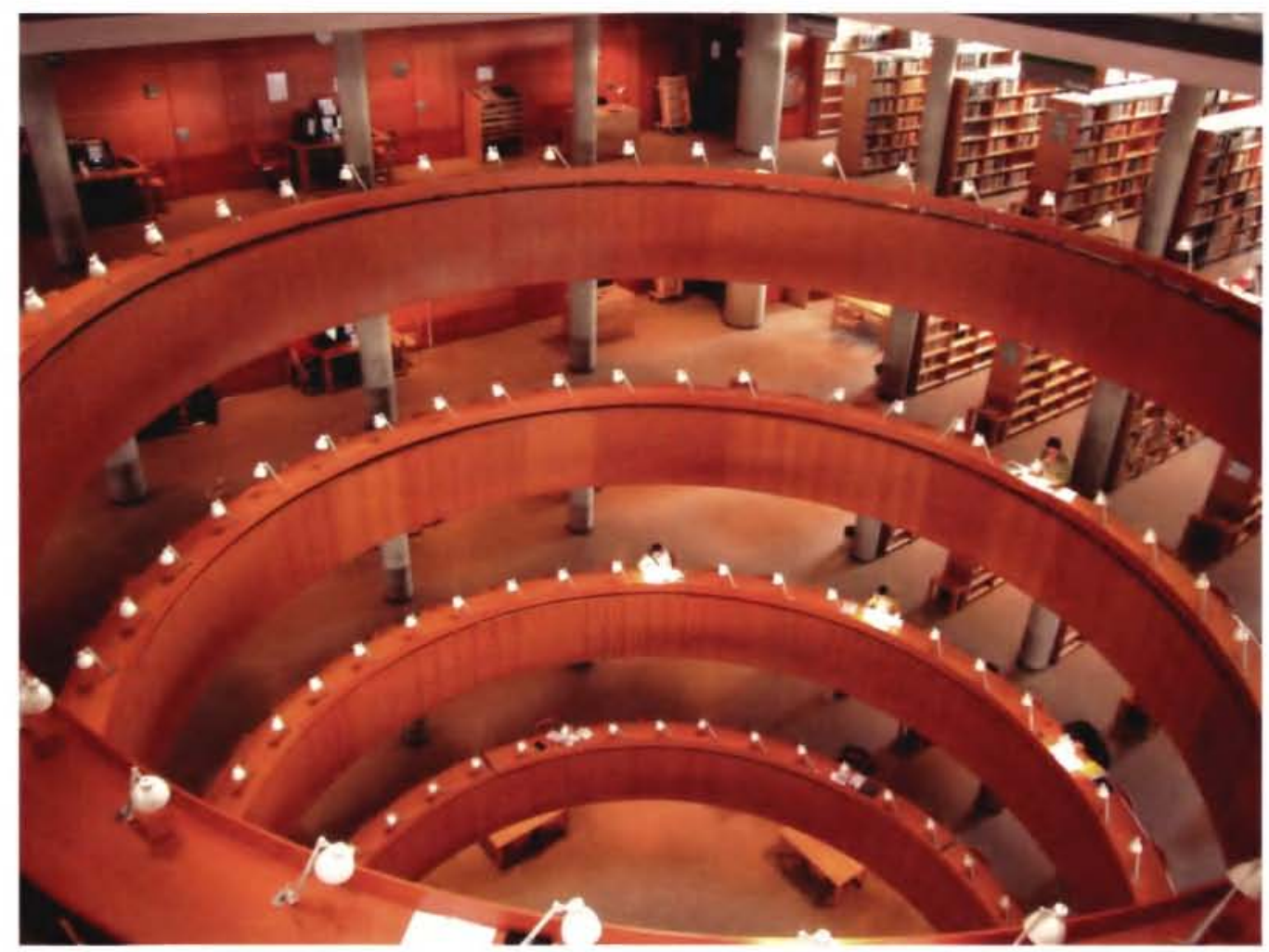

10.1515/ABITECH.2010.30.2.100 


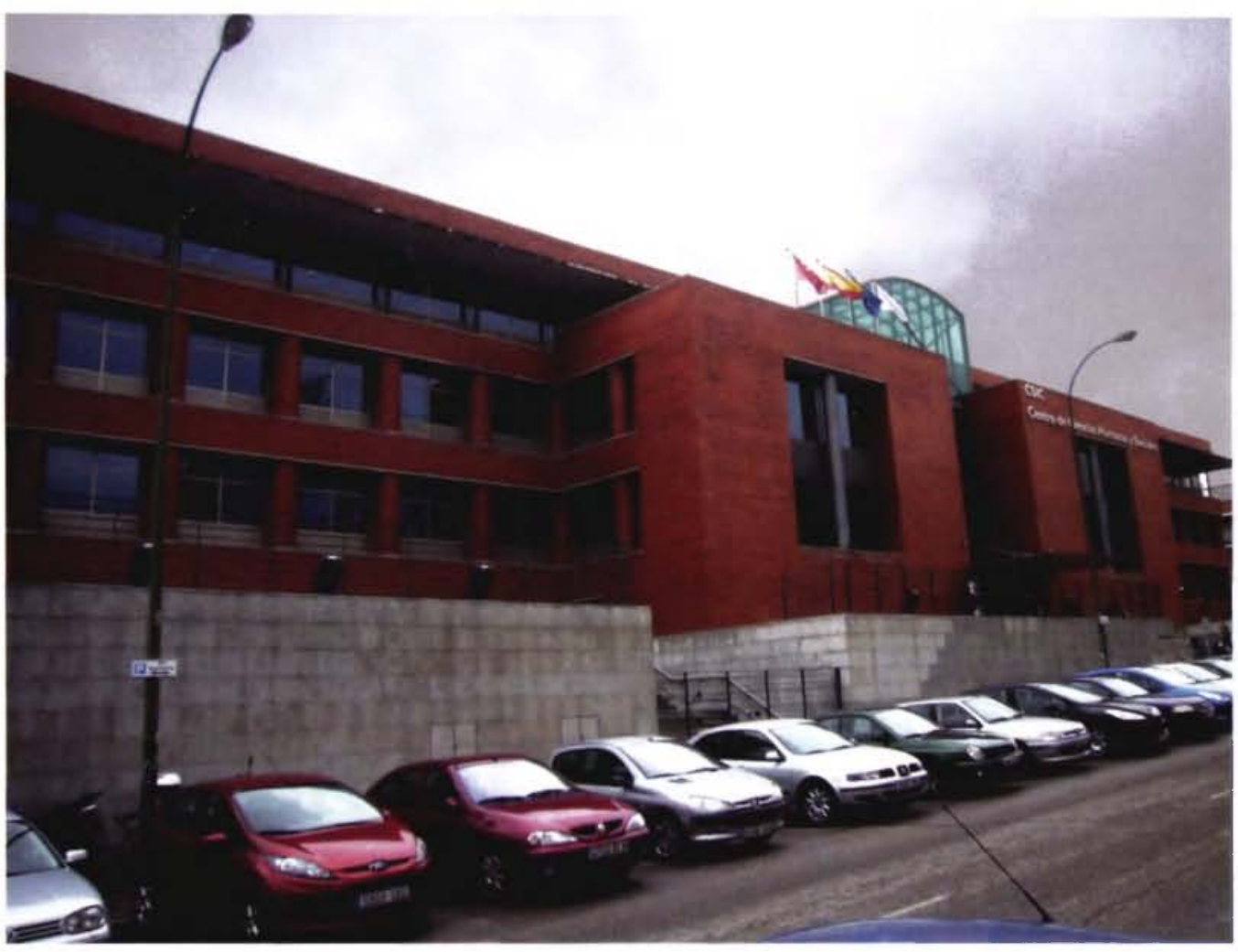

Biblioteca Tomás Navarro Tomás del Consejo Superior de Investigaciones Cientificas (CSIC), Madrid [33]

Centre for Human and Social Sciences CCHS - CSIC Spanish National Research Council

Zirka $20.000 \mathrm{~m}^{2}$, Umbau 2000 bis 2005

Leseplätze: 212

Architekt: Antonio Fernandez Alba [34]

Adresse: Calle de Albasanz, 26-28.

28037 Madrid

Metro: Ciudad Lineal

Die Bibliothek ist Teil des spanischen Forschungsinstituts "Consejo Superior de Investigaciones Cientificas" (CSIC) mit dem Schwerpunkt Geistes- und Sozialwissenschaften (Abb, 19). Dazu wurden sieben Forschungseinrichtungen zusammengelegt, so dass heute über 700 Mitarbeiter beschäftigt sind. Die zentrale Bibliothek ist für 66 Forschergruppen besonders wichtig und durch die Vertretung im Board des Zentrums auch nah an den Entwicklungen in der Forschung. Das Gebäude war in den $80^{\prime}$ er Jahren ursprünglich für eine Lotterie errichtet worden. Die Sanierung bzw. der Umbau wurden 2005 beendet. Der zentrale Saal für die Übertragung der Ziehung im Fernsehen ist heute Lesesaal mit Arbeitsplätzen (Abb. 20). Ein hoher Anteil Freihandliteratur und RFID begünstigen die Selbstbedienung der Forscher. Im Publikumsbereich wurde überall Hohlraumfußboden eingebaut, mit dem negativen Nebeneffekt, dass der Fußboden als Resonanzkörper wirkt. Die Sammlung umfasst zirka 700.000 Bücher, 10.300 Zeitschriftentitel und 11.000 Karten.

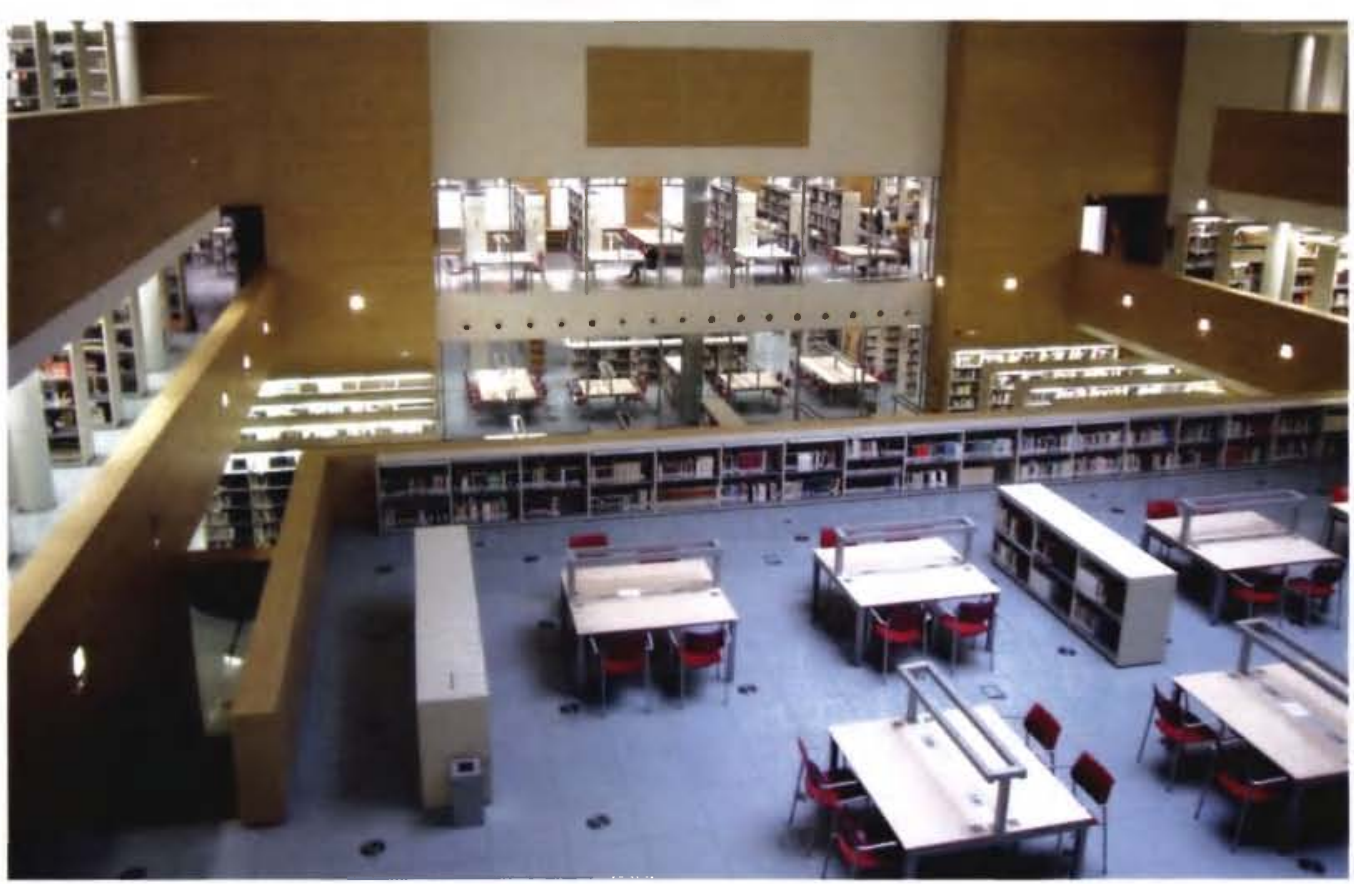

Abb. 19:

Biblioteca Tomás Navarro, CCHS, Ansicht, Foto: Feldsien
Abb. 20

Biblioteca Tomás Navarro, CCHS, zentraler Lesesaal, Foto: Feldsien 
○ 15. Seminar der LIBER Architecture Group

Abb. 21:

Biblioteca Pública de Guadalajara-Biblioteca de Dávalos, Ansicht, Foto: Feldsien

$A b b .22$ :

Biblioteca de Dávalos, zentraler Raum,

Foto: Feldsien

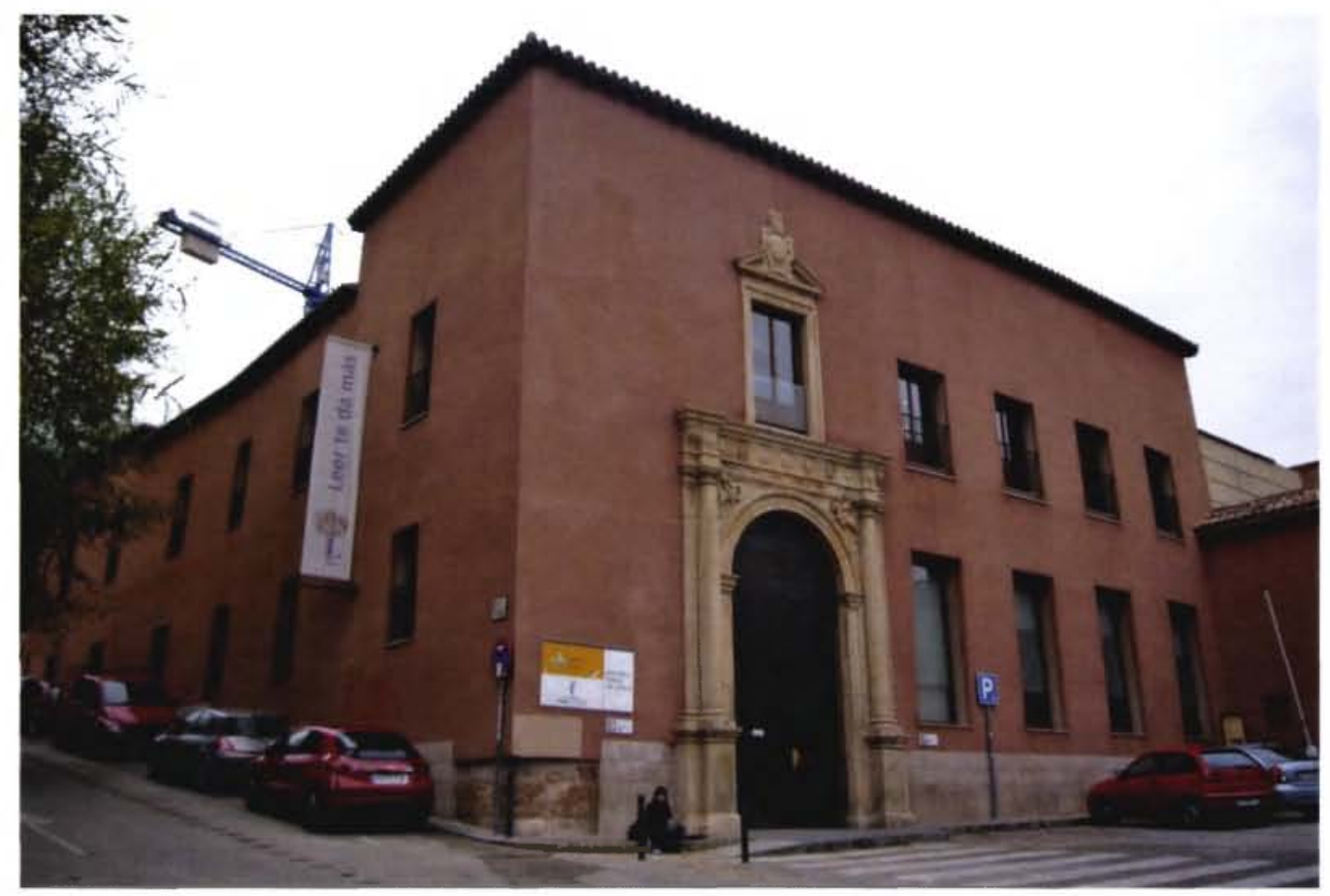

Biblioteca Publica de Guadalajara -

\section{Biblioteca de Dávalos [35]}

$5.852 \mathrm{~m}^{2}$, sechs Millionen Euro Baukosten, Eröffnung 2005

Architekten: Francisco Fernandez Longoria, Dionisio Hernandez Gil

Adresse: Plaza de Dávalos s/n.

19001 Guadalajara

Leseplätze: 223

Die Bibliothek nutzt einen alten Palast aus dem 16. Jahrhundert, der aus Lehm errichtet ist (Abb. 21). Alte bemalte Holzdecken sind zum Teil noch erhalten bzw. wurden detailgetreu ergänzt. Neben einer beeindruckenden Eingangshalle dominiert im Erdgeschoss ein zentraler Hof, der mit Steinsäulen aus dem alten Palazzo umgeben ist (Abb. 22). Den Flügel im zentralen Hof darf jeder Besucher benutzen, der gut Klavier spielen kann. Zeitweise erklingt eine Klavieruntermalung durch das Haus, die sehr gut zu dem denkmalgeschützten Ambiente passt. Der restaurierte alte Palast hat eine moderne Erweiterung erhalten. Insgesamt sind zirka 100.000 Medien verfügbar.

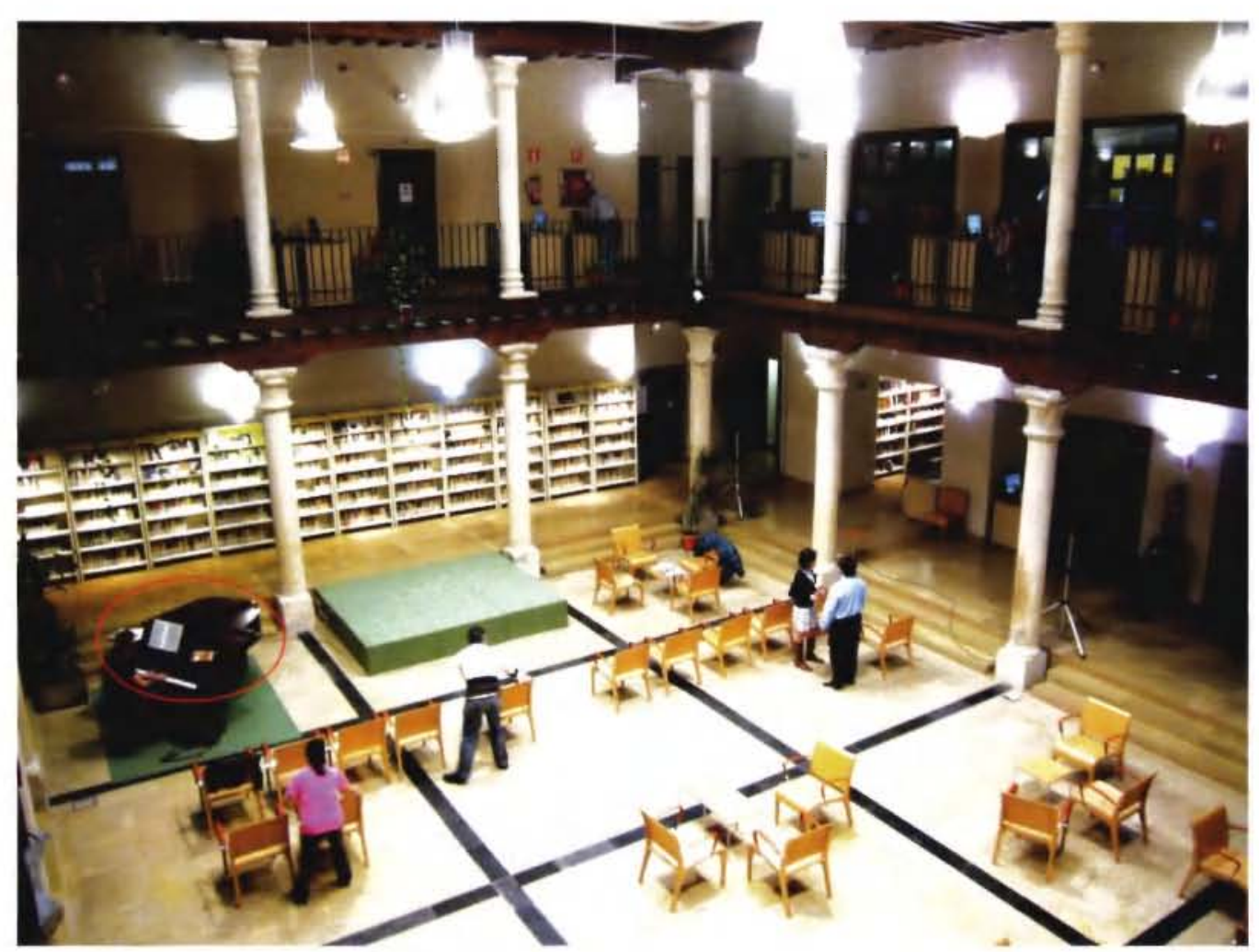




\section{Literatur:}

Alle Vorträge des Seminars sind zugänglich über: http://www.zhbluzern.ch/LIBER-LAG/lageps. htm\#madrid

[1] IBBT= Interdisciplinary Institute for Broad Band Technology, http://www.ibbt.be/en/ibbt

[2] http://foursquare.com/

[3] Das Gartner-Orakel: Die 10 strategischen ITTrends 2010 / Werner Kurzlechner, Thomas Pelkmann. In: Cio, 08.01.2010 http://www.cio de/strategien/methoden/2212689/index.html

Gartner Outlines 10 Mobile Technologies to Watch in 2010 and 2011. http://www.gartner.com/it/page.jsp?id $=1328113$

[4] http://bigtrends.blog.de/2010/04/12/financialtimes-nutzt-foursquare-werbepartner-8351306/

[5] http://www.ted.com/index.php/talks/pattie maes_demos_the_sixth_sense.html

[6] http://www.dekrook.be/ bzw. http://www.ibbt. be/en/nieuws/exhibition-de-krook-new-beacon-ghent-0

[7] http://www.netzpiloten.de/2010/04/14/tedtalkswhat-adults-can-learn-from-kids/

[8] http://www.mediatheques-cus.fr/

[9] http://www.ibosvitart.com/

[10] http://www.techlib.cz/en/

[11] http://www.univ-paris-diderot.fr/sc/site.php

[12] Nationalbibliothek von Spanien

[13] http://www.junqueraarquitectos.com/

[14] http://www.robbrechtendaem.com/

[15] Video über das Delfter Projekt: Multitouch Microsoft Surface: Cultural Heritage Browser from Jaap van de Geer on Vimeo. http://vimeo.com/ 5643953

Forschungen im Bereich Multitouch-Screen etc.: http://www.hpi.uni-potsdam.de/baudisch/ projects/lumino.html;

http://www.patrickbaudisch.com/publications/ 2010-Baudisch-CHI10-Lumino.mp4

Beispiel für Anwendungen: Audi Car Configurator on Surface @ IAA 2009 by Neue Digitale / Razorfish: http://www.vimeo.com/6796111

[16] http://www.gallifordtry.co.uk/ http://www.fcbstudios.com/

[17] http://www.breeam.org

[18] Architekten Dissing + Weitling: http://www.dw.dk/dk/projekter/det-humanistiske-fakultetsbibliotek.aspx
[19] Architekten Atkins Design Studio http://www.atkinsdesign.com/html/ projects_education_british.htm

[20] s.a. Präsentation im Rahmen der IFLA PAC 5/6. Juni 2009 in Paris: „In and Out Air Strategies. From Climate Change to Microclimate. Library. Archives and Museum Preservation Issues": British Library Low Oxygen Case Study. The Future of Fire Prevention. in: Archival Storage von John de Lucy www.ifla.org/files/pac/ British-Library-low-oxygen-case-study.pdf

[21] Broschüre über den Neubau in Leicester: www2.le.ac.uk/offices/press/media-centre/thedavid-wilson-library-1/Building_DW_Library.pdf

[22] http://www.maxdudler.com/43-0-Jacob-undWilhelm-Grimm-Zentrum.html

[23] http://sacm.jccm.es/biblioteca_regional/ biblioteca_discapacitados/informacion_general/pages/biblioteca.htm

[24] Broschüre ON-SITE TOUR - TOUR F: CIUDAD MUSEO www.aes-multimedia.com/prw/pdf/ tourF.pdf

[25] http://www.madrid.org/bpcm/

[26] Stadtteilbibliothek in Usera, Madrid in: Detail 2005, Heft 3, S. 184-185, 287

[27] Heute zwei Büros: http://www.abalos-sentkiewicz.com. http://www.herrerosarquitectos.com/

[28] http://www.peterhalley.com

[29] http://www-1.munimadrid es/bibliometro/

[30] http://www.madrid.org/bpcm/servlet/ Servidor?opcion $=$ Bibreg

[31] Pläne, Fotos: http://www.librarybuildings.info/spain/regional-library-joaquin-leguina-madrid

[32] http://issuu.com/atencion_usuarios_uned/ docs/visitas

[33] http://bibliotecas.csic.es/bibliotecas/TN.html Video: http://www.youtube.com/ watch? $=$ XbwD4ACPr44 vom 07.02.2008 CSIC: Biblioteca Tomas Navarro Tomas

[34] http://www.soloarquitectura.com/arquitectos/ antonio_fernandez_alba.html

[35] http://www.bibliotecaspublicas.es/guadalajara/ index.jsp

\section{Seminar der $\odot$ LIBER Architecture Group}

Anschrift des Autorin:

Inken Feldsien-Sudhaus Universitätsbibliothek TUHH

Denickestr. 22 21073 Hamburg feldsien-sudhaus@ tu-harburg.de 